\title{
छs \\ First electron-cloud studies at the Large Hadron Collider
}

\author{
O. Domínguez, ${ }^{*}$ K. Li, ${ }^{\dagger}$ G. Arduini, E. Métral, G. Rumolo, and F. Zimmermann \\ CERN, Geneva, Switzerland \\ H. Maury Cuna \\ Departamento de Física Aplicada, CINVESTAV, Unidad Mérida, A.P.73 Cordemex, 97310, Mérida, Yucatán, México
}

(Received 7 August 2012; published 9 January 2013)

\begin{abstract}
During the beam commissioning of the Large Hadron Collider (LHC) [LHC Design Report No. CERN2004-003-V-1, 2004 [http://cds.cern.ch/record/782076?ln=en]; O. Brüning, H. Burkhardt, and S. Myers, Prog. Part. Nucl. Phys. 67, 705 (2012)] with 150, 75, 50, and 25-ns bunch spacing, important electron-cloud effects, like pressure rise, cryogenic heat load, beam instabilities, or emittance growth, were observed. Methods have been developed to infer different key beam-pipe surface parameters by benchmarking simulations and pressure rise as well as heat-load observations. These methods allow us to monitor the scrubbing process, i.e., the reduction of the secondary emission yield as a function of time, in order to decide on the most appropriate strategies for machine operation. To better understand the influence of electron clouds on the beam dynamics, simulations have been carried out to examine both the coherent and the incoherent effects on the beam. In this paper we present the methodology and first results for the scrubbing monitoring process at the LHC. We also review simulated instability thresholds and tune footprints for beams of different emittance, interacting with an electron cloud in field-free or dipole regions.
\end{abstract}

DOI: 10.1103/PhysRevSTAB.16.011003

PACS numbers: $29.20 .-\mathrm{c}$

\section{INTRODUCTION}

For almost 15 years photoemission and secondary emission have been predicted to build up an electron cloud inside the LHC beam pipe [1], similar to the photoelectron instability in positron storage rings [2-4]. The possibility of "beam-induced multipacting" at the LHC had been suggested even earlier [5] extrapolating from observations with bunched beams at the Intersecting Storage Rings in the 1970s [6]. The electron cloud, at sufficiently high density, can cause both single and coupled-bunch instabilities of the proton beam $[1,7]$, give rise to incoherent beam losses or emittance growth [8,9], heat the vacuum chamber (and subsequently provoke a quench in superconducting magnets), or lead to a vacuum pressure increase by several orders of magnitude due to electron stimulated desorption $[9,10]$. All these effects eventually lead to luminosity limitations. From 1999 onward electron-cloud effects have been seen with LHC-type beams first in the Super Proton Synchrotron (SPS), then in the Proton Synchrotron, and finally, since 2010, as expected, in the LHC itself. During the early LHC beam commissioning with 150,75 , and 50-ns bunch spacing important electron-cloud effects, such as

\footnotetext{
*octavio.dominguez@cern.ch

Also at EPFL, LPAP, CH-1015 Lausanne, Switzerland.

${ }^{\dagger}$ Currently at SLAC National Accelerator Laboratory, 2575 Sand Hill Road, Menlo Park, CA 94025-7015, USA.

Published by the American Physical Society under the terms of the Creative Commons Attribution 3.0 License. Further distribution of this work must maintain attribution to the author(s) and the published article's title, journal citation, and DOI.
}

pressure rise, cryogenic heat load, beam instabilities, beam loss, and emittance growth, were observed [11-13]. Several exploratory studies at the design bunch spacing of $25 \mathrm{~ns}$ were also performed during 2011 [14].

The LHC mitigation strategy against electron cloud includes a sawtooth pattern on the horizontally outer side of the so-called beam screen inside the cold arcs, a shield mounted on top of the beam-screen pumping slots blocking the direct path of electrons onto the cold bore of the magnets, Non-Evaporable Getter coating for all the warm sections of the machine, installation of solenoid windings in field-free portions of the interaction region, and, last not least, beam scrubbing, i.e., the reduction of the secondary emission yield (SEY) with increasing electron dose hitting the surface, i.e., as a result of the electron cloud itself. Beam scrubbing is the ultimate mitigation of electroncloud effects of the LHC, and considered necessary to achieve nominal LHC performance [15].

Since dedicated in situ measurements of the LHC electron cloud density and the LHC vacuum-chamber surface properties are not available, we are developing a method to determine the actual surface properties of the vacuum chamber related to secondary emission and to the electron-cloud buildup ( $\delta_{\max }, \varepsilon_{\max }$, and $R$ [16]; see Fig. 1 for a graphical definition of these three quantities), and their evolution in time, based on benchmarking computer simulations of the electron flux on the chamber surface using the ECLOUD code against pressure measurements for different beam characteristics (e.g. for varying spacing between bunch trains). This new method allows monitoring the effectiveness of LHC "scrubbing runs" and provides 


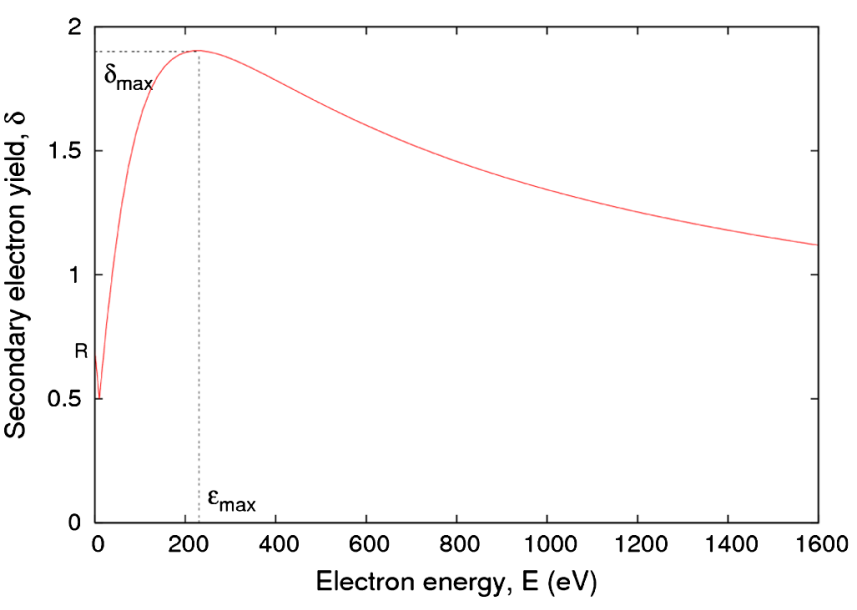

FIG. 1. Secondary emission yield as a function of primary electron energy, defining the parameters $\delta_{\max }, \varepsilon_{\max }$, and $R$.

snapshots of the surface conditions around the LHC ring. A similar approach has been adopted for the cold arcs, where unexpectedly accurate measurements of the cryogenic heat load [17] can be used to benchmark electron-cloud simulations of the electron energy deposited on the so-called beam screen inside the superconducting magnets.

In parallel, a simulation campaign has been launched to estimate the threshold values for coherent instabilities induced by electron clouds in the LHC in order to improve the understanding of the impact of the electron cloud on the beam dynamics and to determine for which surface parameters the beam will be stable. Threshold values have been determined for straight sections and for bends at flatbottom $(450 \mathrm{GeV})$ and at the present flattop $(3.5 \mathrm{TeV})$ energy and for different transverse normalized emittances. The simulations were performed with the code HEADTAIL $[21,22]$.

\section{PRESSURE BENCHMARKING FOR THE LHC WARM SECTIONS}

At injection energy $(450 \mathrm{GeV})$, the pressure inside the vacuum beam pipe affects the speed of the electron-cloud buildup, since the initial electrons are produced by gas ionization. However, if there is noticeable multipacting, the rate of primary electrons does not significantly influence the final value of the saturated electron density, which is then determined by secondary emission (multipacting) and by the space-charge field of the electron cloud itself. In such a case, larger vacuum pressures just make the electron density reach its equilibrium value faster. This is due to the fact that the energy spectrum of electrons hitting the wall is insensitive to the pressure [23].

Nevertheless, in order to infer the best estimates of the beam-pipe characteristics, the steady-state vacuum pressure of the machine at each stage of the experiment has to be introduced as an input parameter in the simulations in order to correctly take into account the multiturn nature of the pressure evolution in a circular accelerator like the LHC. This is due to the fact that the time constant of the vacuum evolution is much longer than the revolution period, while the electron-cloud buildup simulations typically model only a fraction of a turn. According to the vacuum-gauge measurements, a steady-state pressure is normally established a few minutes after injecting the last bunch train for a given configuration.

Assuming that the pressure increase is proportional to the electron flux hitting the chamber wall, pressure measurements for different bunch train configurations (e.g. with changing spacing between trains or with a varying number of trains injected into the machine) can be benchmarked against simulations by comparing ratios of observed pressure increases and of simulated electron fluxes at the wall, respectively. The idea of the benchmarking using ratios goes back to an earlier study for the SPS (serving as LHC injector) where the electron-cloud flux could be measured directly [24]. In the LHC case, no electron-cloud monitor is available, but instead the measured increase in the vacuum pressure is taken to be a reliable indicator proportional to the electron flux on the wall.

\section{A. Methodology}

We face a four-parameter problem. The steps followed in the benchmarking are the following: (1) We fix two of the parameters, namely the pressure (using the measured value) and $\varepsilon_{\max }$ (set to $230 \mathrm{eV}$, which seems to be a good first estimate according to past surface measurements and some previous simulation benchmarking [25]). (2) We simulate the electron-cloud buildup for different bunch configurations using the ECLOUD code, scanning the other two parameters, $\delta_{\max }$ and $R$, in steps of 0.1 and 0.05 , respectively. Smaller steps introduce statistical noise which needs to be controlled by smoothing techniques. (3) For each bunch configuration we plot the simulated electron flux $\phi_{i}$ above a 2D grid spanned by $\delta_{\max }$ and $R$. (4) We fit the flux simulated on the grid to a third order polynomial and then form the ratio of simulated fluxes (that is, dividing the polynomials) for two different bunch configurations (the fluxes and not their ratio are fitted in order to suppress the effect of statistical fluctuations). (5) Comparing the latter ratio with the experimental ratio of measured pressure increases yields a curve in the $\delta_{\max } R$ plane (see Fig. 2). Different configurations yield different curves in that plane. (6) If the measurements contain sufficient information and the simulation model is reasonably accurate, we expect to obtain a unique intersection between lines corresponding to different bunch configurations. This crossing point then defines the solution for $\delta_{\max }$ and $R$.

\section{B. Results}

Until now we have processed four sets of measurements obtained during the conditioning of the machine through beam scrubbing. All of these have been recorded at a beam 


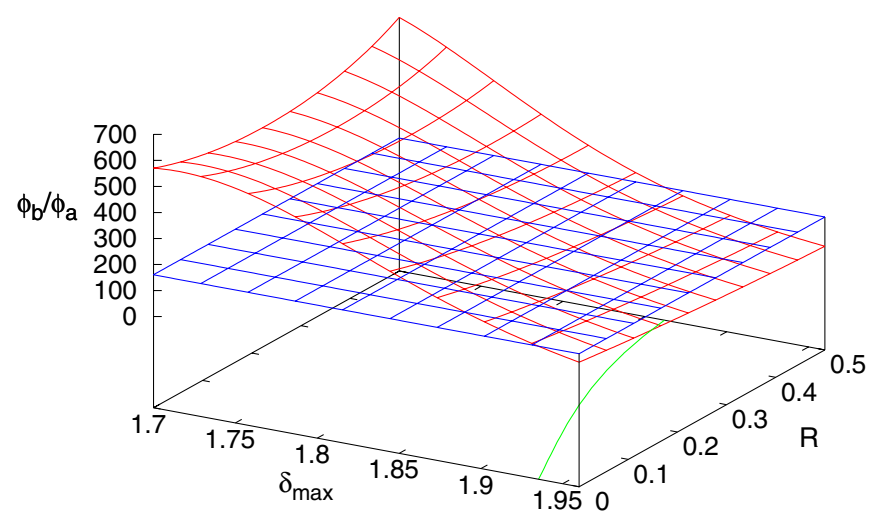

FIG. 2. Example of a 3D surface of simulated fluxes for the case $\phi_{b} / \phi_{a}$ (red) cut by a plane surface (blue) at the value equal to the ratio of the corresponding measured pressures $\left(P_{b} / P_{a}=\right.$ 161.0). The bottom plane shows the contour of the intersection between both surfaces (green).

energy of $450 \mathrm{GeV}$ with 50 and $25 \mathrm{~ns}$ bunch spacing for the first three sets and the last set, respectively. In the benchmarking simulations the geometry of the vacuum chamber is taken to be round (with $40 \mathrm{~mm}$ radius), as in the modules hosting the pressure gauges. We only present results for one ionization gauge. Results for other gauges look similar. We have used two kinds of beam configurations, with varying spacing between successive bunch trains (also called "batches") and varying number of batches, respectively. Table I lists the parameters for the three sets of measurements with $50 \mathrm{~ns}$ bunch spacing.

At the beginning of a scrubbing run in April 2011, two experiments were carried out (both corresponding to set 1 listed above). In the first we injected batches in pairs with varying batch spacing ( $6 \mu \mathrm{s}, 4 \mu \mathrm{s}$, and $2 \mu \mathrm{s}$ ). Each pair of batches is separated by $11.5 \mu \mathrm{s}$ (time enough to kill any electron cloud). Figure 3 shows the pressure increases observed during this first experiment, including the first 12-bunch batch (where no pressure increase can be appreciated). In the second experiment we injected an increasing number of batches at a batch-to-batch distance of $2.125 \mu \mathrm{s}$ (up to 5). Figure 4 depicts the results obtained for both experiments. We could conclude that the solution is around

TABLE I. Parameters used in the simulations for the different sets of measurements with $50 \mathrm{~ns}$ bunch spacing. The value $0.225 \mu \mathrm{s}$ for the batch spacing in sets 2 and 3 refers to the space between two or three trains of 36 bunches injected simultaneously.

\begin{tabular}{|c|c|c|c|}
\hline & Set 1 & Set 2 & Set 3 \\
\hline $\begin{array}{l}\text { Number of bunches } \\
\text { per batch }\end{array}$ & 36 & $72(2 \times 36)$ & $108(3 \times 36)$ \\
\hline Number of batches & $1-5$ & $1-14$ & $1-12$ \\
\hline Batch spacing $(\mu \mathrm{s})$ & $2.0,4.0,6.0$ & $0.225,4.850$ & $0.225,0.925$ \\
\hline $\begin{array}{l}\text { Average bunch } \\
\text { population }\left(10^{11} \mathrm{ppb}\right)\end{array}$ & 1.1 & 1.21 & 1.15 \\
\hline
\end{tabular}

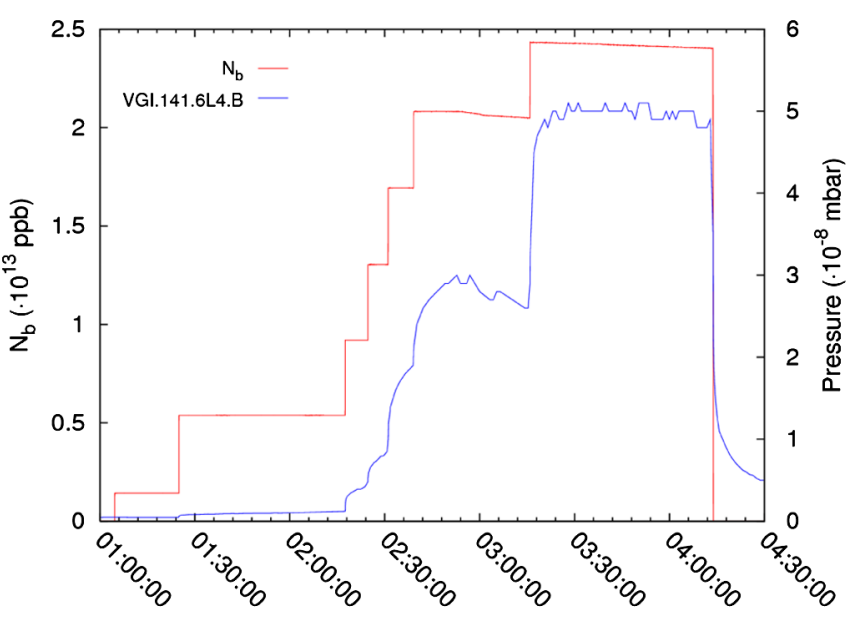

FIG. 3. Beam intensity and pressure at the gauge VGI.141.6L4.B during the first experiment on 6 April 2011. Every step in the beam intensity (red curve) indicates the injection of a new batch.

$\delta_{\max }=1.9$ and $R=0.2$. We have to take into account that there are large uncertainties in the measured pressure values as well as in the estimated bunch population. According to simulations, such uncertainties can lead to a mismatch between lines and prevent a single unique intersection, as illustrated by this example. The value of $\delta_{\max }=1.9$ is in agreement with some previous estimations from the VSC group at CERN, who gave a value between 1.6 and $1.9[27,28]$. In addition, the value $R=0.2$ is in agreement with several high precision measurements, both recent (e.g. [29]) and old (e.g. [30]).

After a few days of surface conditioning, double batches of 36 bunches each separated by $225 \mathrm{~ns}$ were injected at a distance of $4.85 \mu \mathrm{s}$ (up to 14). This corresponds to set 2 of the experimental data. A similar experiment (set 3) took place in mid-May 2011 but using triple batches instead,

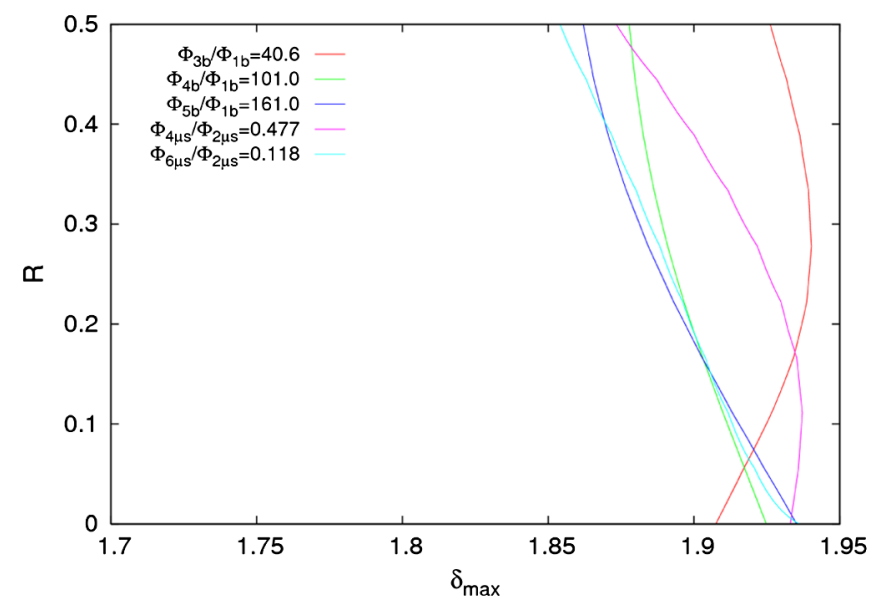

FIG. 4. Combinations of $\delta_{\text {max }}-R$ values characterizing the chamber surface, obtained by benchmarking ratios of observed pressure increases against ratios of simulated electron fluxes, for measurements on 6 April 2011. 

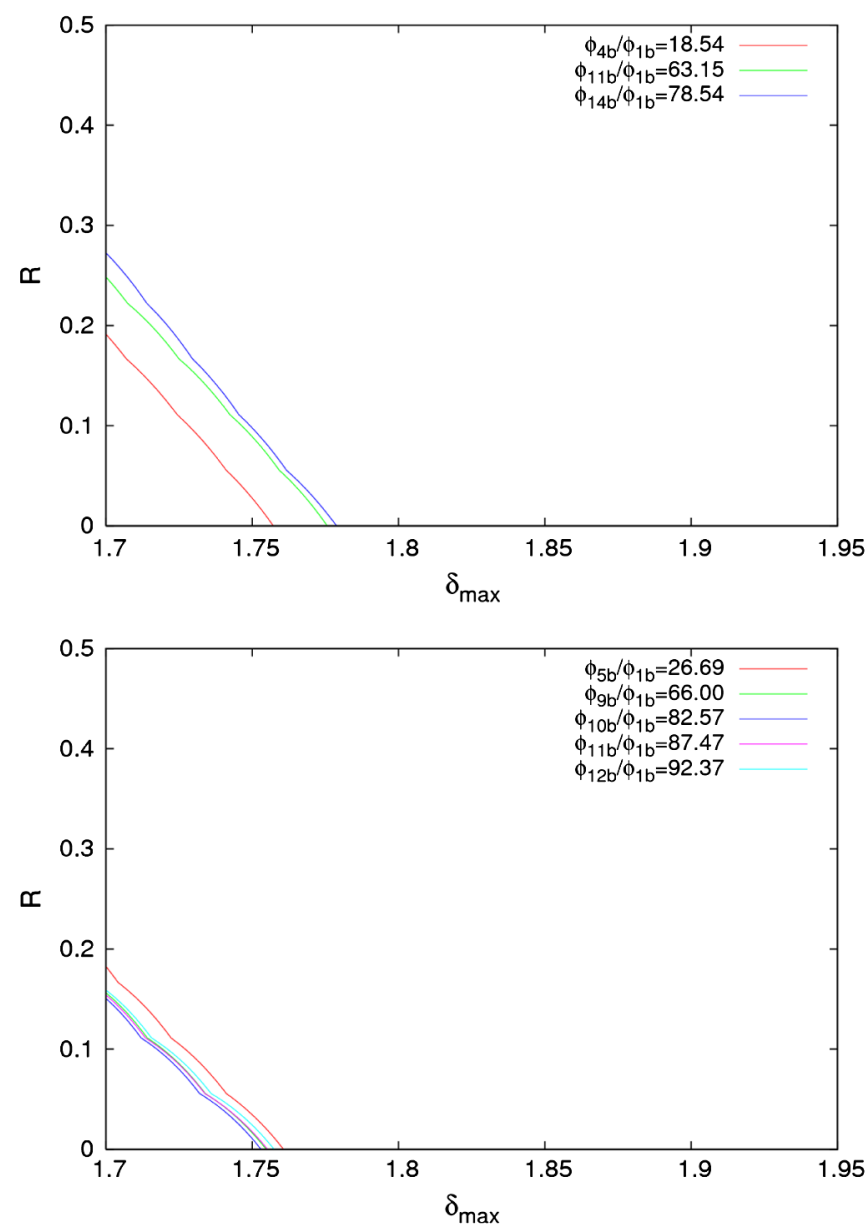

FIG. 5. Combinations of $\delta_{\max }-R$ values characterizing the chamber surface, obtained by benchmarking ratios of observed pressure increases against ratios of simulated electron fluxes, for measurements on 11 April (top) and 19 May 2011 (bottom).

again separated by $225 \mathrm{~ns}$, at a distance of $925 \mathrm{~ns}$ (up to 12). Figure 5 shows the results obtained in these cases. It is worth noting that for these last two cases we observe parallel lines instead of a clear cut between the lines. This is due to the loss of memory from the 225 ns gap between 36bunch batches that appears when we inject the double (or triple) batches together. Indeed the lines should be the same in theory. The conclusion is that it is necessary to have two sets of measurements during the same experiment with different batch spacings in order to obtain lines of different slope which uniquely intersect. The intersection between sets of lines would yield the desired solution.

TABLE II. Parameters used in the simulations for the measurements with $25 \mathrm{~ns}$ bunch spacing.

\begin{tabular}{lc}
\hline \hline & Set 4 \\
\hline Number of bunches per batch & 72 \\
Number of batches & 2 \\
Batch spacing $(\mu \mathrm{s})$ & $1.0,2.0,3.0,4.0$ \\
Bunch population $\left(10^{11} \mathrm{ppb}\right)$ & $1.1,1.0,1.0,1.1$ \\
\hline \hline
\end{tabular}

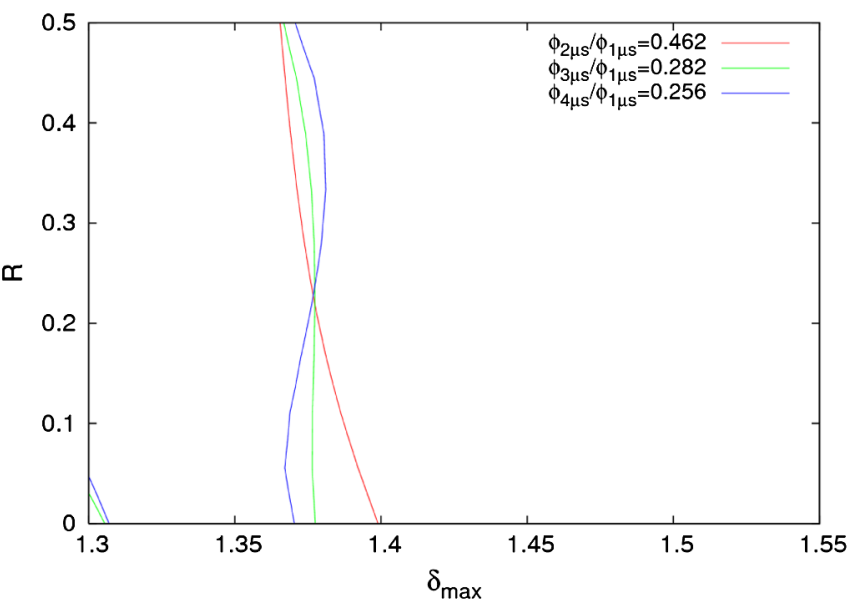

FIG. 6. Combinations of $\delta_{\max }-R$ values characterizing the chamber surface, obtained by benchmarking ratios of observed pressure increases against ratios of simulated electron fluxes, for measurements on 25 October 2011. In this case a value $\varepsilon_{\max }=$ $260 \mathrm{eV}$ has been assumed, as it gives a better fit to the data. This could be a sign of variation of $\varepsilon_{\max }$ during the scrubbing.

A new measurement, with varying spacing between batches, has been carried out at the end of the 2011's proton run. In this occasion the bunch spacing was reduced to $25 \mathrm{~ns}$. Table II shows the parameters used in this case and Fig. 6 depicts the result obtained for this experiment, which reflects the latest, most conditioned, state of the machine to date since only a few proton physics runs took place after these experiments and ion physics runs do not produce any electron-cloud evidence.

Although we are not yet able to extract a unique value for $\delta_{\text {max }}$ and $R$, we can clearly see evidence for conditioning, as the solution for later cases tends towards lower $\delta_{\max }$ values. In addition, the evolution of the conditioning for 50-ns and 25-ns beams looks as expected, with $\delta_{\max }$ approaching the 50-ns or 25-ns threshold, respectively, as asymptotic limit (see Fig. 26). All these facts instill some confidence in the method and support its potential use as a tool for monitoring the surface conditioning through beam scrubbing.

\section{HEAT-LOAD BENCHMARKING FOR THE COLD ARCS}

The beam-screen heat load due to resistive wall (image currents), synchrotron radiation, and electron cloud depends strongly on the beam energy, the bunch intensity, as well as the number and length of the circulating bunches [31]. An individual beam-screen cooling loop extends over $53 \mathrm{~m}$ (half an optical cell). Along this region there are dipoles, quadrupoles, and short drift sections. Because of some computing limitations, in this paper we report simulation results for dipoles and drift sections, i.e., we present average heat loads per unit length for a reduced half optical cell of $49.3 \mathrm{~m}$ length (that is, excluding the quadrupoles from the calculation). 
TABLE III. Simulation parameters for sets A, B, and C (for B only an energy of $3.5 \mathrm{TeV}$ with 50 -ns bunch spacing is considered, whereas for $\mathrm{C}$ only injection energy with 25-ns bunch spacing is simulated).

\begin{tabular}{lcc}
\hline \hline Parameter & $450 \mathrm{GeV}$ & $3.5 \mathrm{TeV}$ \\
\hline Bunch intensity $\left(10^{11} \mathrm{ppb}\right)$ & 1.1 & 1.2 \\
Primary photoelectron emission yield & $\cdots$ & $1.233 \times 10^{-4}$ \\
Bunch length $(\mathrm{cm})$ & 11.8 & 9 \\
$\sigma_{x}=\sigma_{y}(\mathrm{~mm})$ & 1.2 & 0.3 \\
Pressure (mbar) & $4.3 \times 10^{-8}$ & $\cdots$ \\
\hline \hline
\end{tabular}

\section{A. Methodology}

A first set of simulation (called set A from now on) was launched in order to determine the multipacting thresholds at injection and top energy for $50 \mathrm{~ns}$ bunch spacing. For this set we used the simulation parameters shown in Table III and a filling pattern consisting of eight batches of 36 bunches each, with a batch spacing of 2.125 us. All the simulations were performed for a Gaussian bunch profile.

During the scrubbing run in 2011 at $50 \mathrm{~ns}$ bunch spacing, an important electron cloud related heat load was measured. This motivated a second set of simulations (set B) at 3.5 TeV beam energy, with the same parameters as for the previous one (see Table III). In addition, for this set we changed the filling scheme from the previous one and we simulated the bunch filling pattern from LHC fill No. 1704 (13/4/2011) with the following filling scheme (for both beams): 228 bunches per beam with an average intensity of $1.22 \times 10^{11}$ protons per bunch. We omitted the pilot bunches and the small intermediate 12-bunch batches, which are not expected to contribute to the electron-cloud buildup, and we simulated the last six batches of 36

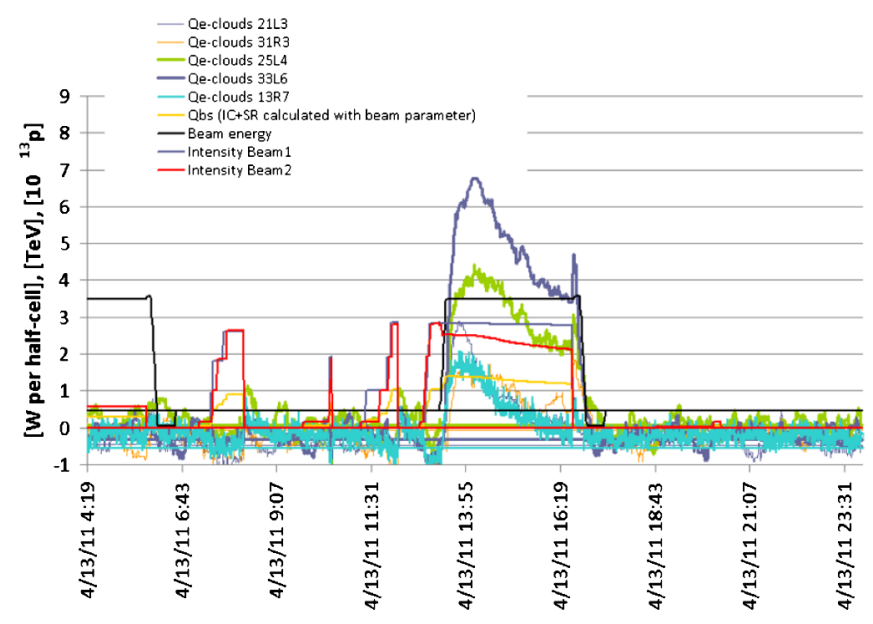

FIG. 7. Measured heat load due to the electron cloud during the scrubbing process at $50 \mathrm{~ns}$ at a beam energy of $3.5 \mathrm{TeV}$ on 13 April 2011. Qbs refers to the computed heat load on the beam screen due to image currents and synchrotron radiation, whereas $Q e$ clouds are the heat loads associated to electron cloud in different locations, obtained by subtracting $Q b s$ from the measured values.
TABLE IV. Summary of simulation parameters for set D.

\begin{tabular}{|c|c|c|c|}
\hline Parameter & \multicolumn{3}{|c|}{$3.5 \mathrm{TeV}$} \\
\hline Bunch intensity (ppb) & \multicolumn{3}{|c|}{$1.15 \times 10^{11}$} \\
\hline SEY & \multicolumn{3}{|c|}{$1.0-3.0$} \\
\hline Primary photoelectron emission yield & \multicolumn{3}{|c|}{$1.233 \times 10^{-4}$} \\
\hline$R$ & \multicolumn{3}{|c|}{$0.0-1.0$} \\
\hline Bunch length $(\mathrm{cm})$ & \multicolumn{3}{|c|}{9} \\
\hline$\sigma_{x}=\sigma_{y}(\mathrm{~mm})$ & \multicolumn{3}{|c|}{0.3} \\
\hline Bunch spacing (ns) & 25 & 50 & 75 \\
\hline Number of bunches per batch & 72 & 36 & 24 \\
\hline Batch spacing (ns) & 200 & 200 & 225 \\
\hline
\end{tabular}

bunches of this scheme (216 bunches in total). Between the two batches of a pair there was a spacing of $225 \mathrm{~ns}$ (in the simulations $200 \mathrm{~ns}$ have been considered due to computational limitations) and between pairs a spacing of $1.1 \mu \mathrm{s}$. The measured data are shown in Fig. 7, which present the total electron-cloud heat load per half cell. To obtain the heat load per beam and per meter, the values shown have to be divided by about a factor of 100 . Then, from Fig. 7, the average heat load due to electron cloud (which depends on location) is of the order of $40-50 \mathrm{~mW} / \mathrm{m} /$ beam. The measurement resolution is estimated to be about $5-10 \mathrm{~mW} / \mathrm{m} /$ beam.

A third set of simulations (set C) was executed in order to benchmark simulations against a measured heat-load value of approximately $1 \mathrm{~W} / \mathrm{m}$ in October 2011 at injection energy and $25 \mathrm{~ns}$ bunch spacing. We also included a study of the electron volume density for this case (see Fig. 28). We again used the parameters listed in Table III with a slightly different SEY range (1.5 to 2.0). The filling pattern consisted of batches of 72 bunches, with a batch separation of $925 \mathrm{~ns}$.

The fourth set of simulations (set D) was a study of the multipacting thresholds as a function of the reflectivity $R$

TABLE V. Summary of multipacting thresholds for a dipole section of sets A and B (50-ns bunch spacing).

\begin{tabular}{lcc}
\hline \hline Energy & Reflectivity & SEY \\
\hline & 0.2 & 2.4 \\
$450 \mathrm{GeV}$ & 0.3 & 2.3 \\
& 0.4 & 2.2 \\
& 0.5 & 2.1 \\
& 0.6 & 2.0 \\
& 0.2 & 2.3 \\
& 0.3 & 2.2 \\
$3.5 \mathrm{TeV}$ & 0.4 & 2.1 \\
& 0.5 & 2.0 \\
& 0.6 & 1.9 \\
& 0.7 & 1.8 \\
& 0.8 & 1.7 \\
& 0.9 & 1.6 \\
\hline \hline
\end{tabular}




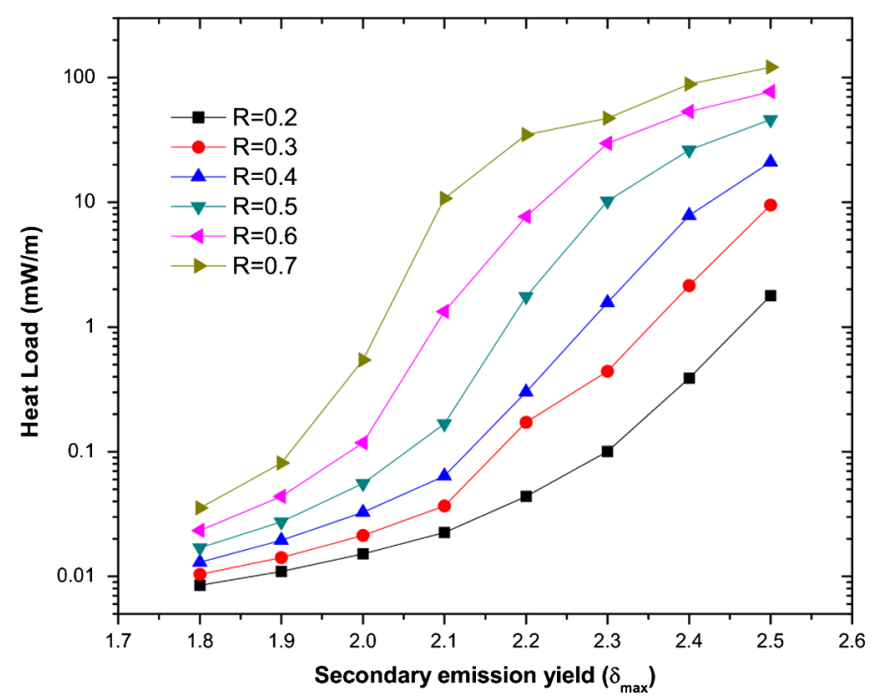

FIG. 8. Simulated average arc heat load for $50 \mathrm{~ns}$ bunch spacing at injection energy. Bunch population: $1.1 \times 10^{11} \mathrm{ppb}$. During the corresponding experiment, an average heat load of about $20 \mathrm{~mW} / \mathrm{m}$ was measured.

and the bunch spacing. In Table IV, we list the parameters used for these simulations.

In order to benchmark simulations and measurements we have used the different sets in order to infer the multipacting thresholds for the different situations and to generate contour plots that allow us to pin down the values of $\delta_{\max }$ that are compatible with heat-load observations. For copper, values of $R$ between 0.2 and 1.0 can be found in the literature (e.g. [16,32]). In general, we present a scan over $R$ and the corresponding value of $\delta_{\max }$. However,

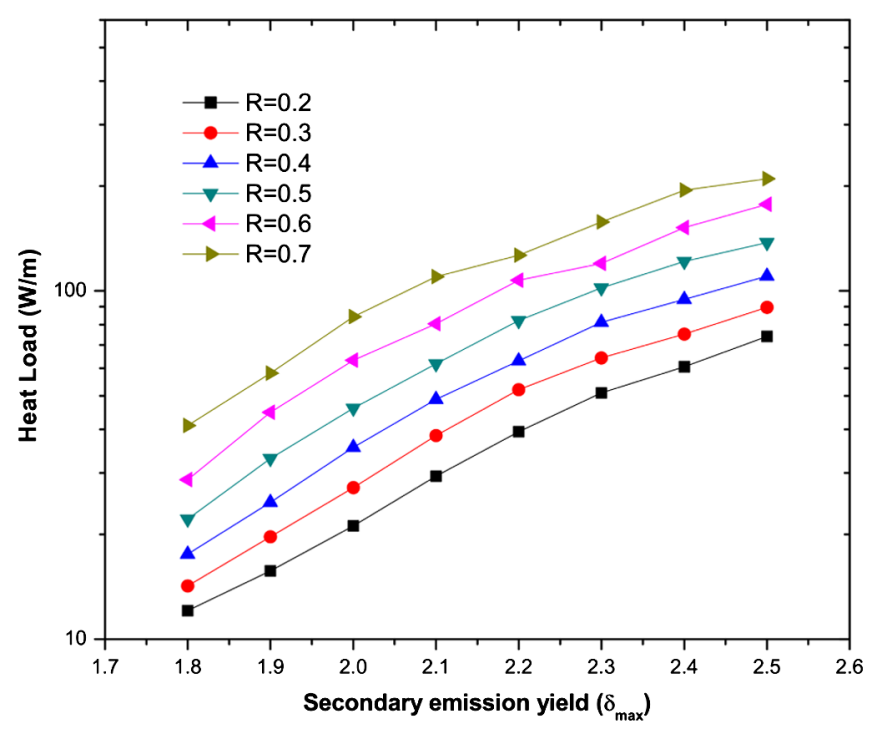

FIG. 9. Simulated average arc heat load for $50 \mathrm{~ns}$ bunch spacing at $3.5 \mathrm{TeV}$. Bunch population: $1.2 \times 10^{11} \mathrm{ppb}$. During the corresponding experiment, an average heat load of about $45 \mathrm{~mW} / \mathrm{m}$ was measured.

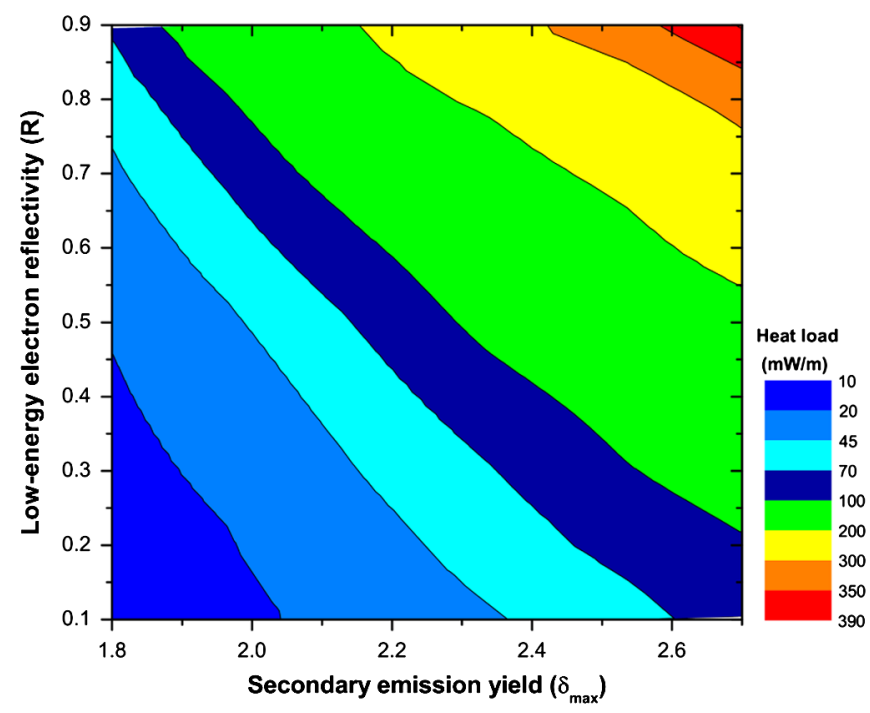

FIG. 10. Heat-load contour plot for $50 \mathrm{~ns}$ bunch spacing at $3.5 \mathrm{TeV}$.

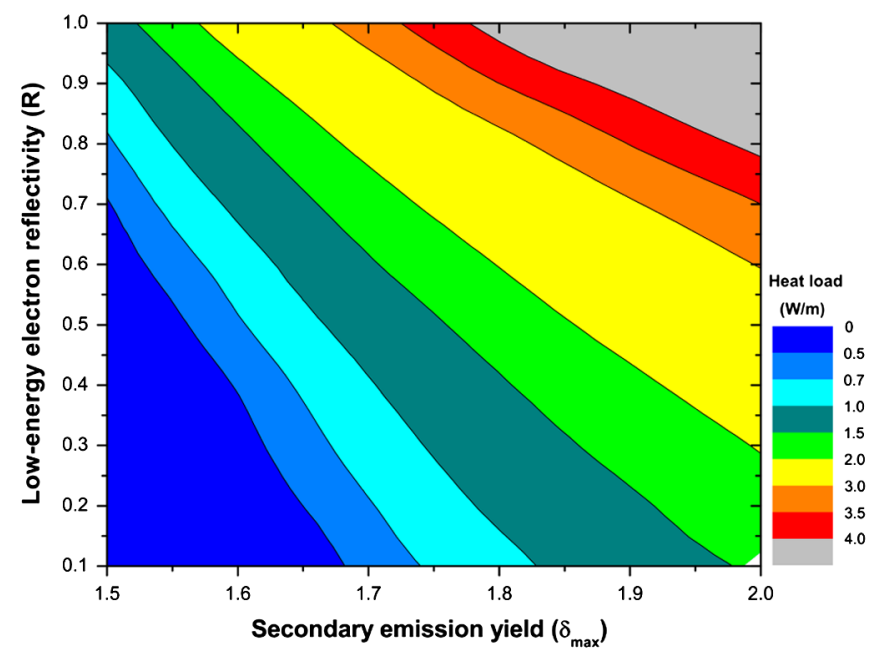

FIG. 11. Heat-load contour plot for $25 \mathrm{~ns}$ bunch spacing at $450 \mathrm{GeV}$.

taking into account measurements for an LHC dipole chamber prototype [32,33], we here consider a fixed value $R=0.5$ to estimate the heat load in the LHC arcs and then pin down the corresponding $\delta_{\max }$ value.

\section{B. Results}

Table V shows the multipacting thresholds found from the simulations of set A and set B. We notice that there is not a very big difference in the thresholds between the $450 \mathrm{GeV}$ injection energy and $3.5 \mathrm{TeV}$ beam energy, except for a slightly earlier start of multipacting for the higher energy. We observe a certain correlation between the reflectivity and the multipacting thresholds (i.e. increasing the reflectivity by 0.1 the SEY thresholds decrease by the same amount). 
Figures 8 and 9 present the heat load obtained for simulation set A (averaged over all the simulated batches). We again remark that the difference between the two beam energies is not dramatic, especially for high $\delta_{\max }$ values.

Figure 10 shows a contour plot of the heat load for simulation set B (averaged over all six batches). We can infer that to get the measured heat load of $45 \mathrm{~mW} / \mathrm{m} /$ beam from the 13 April 2011 measurements a maximum secondary emission yield $\left(\delta_{\max }\right)$ slightly above 2.0 is needed. In Fig. 11, we show a contour plot of the heat load for the simulation set $\mathrm{C}$ (averaged over all the simulated batches). We can see that, in this case, to get the measured average heat load around $0.7 \mathrm{~W} / \mathrm{m}$ from the 25 October 2011 measurements a maximum secondary emission yield slightly above 1.6 is needed.

\section{EMITTANCE GROWTH}

Figure 12 shows, as an example, the measured emittance blowup along a group of bunch trains observed on 25 October 2011. As expected if the blowup is due to the electron cloud, the first bunches of each batch are less affected, whereas the last bunches in each train suffer a strongest blowup.

An understanding of the processes giving rise to such blowup is achieved through simulations. Collective effects are often modeled by multiparticle tracking codes. The code HEADTAIL has been chosen as a simulation tool for our study here. It includes a number of different collective effects, is easily extensible, and has been well benchmarked in the past. Recent upgrades of the code, including new data handling and processing, facilitate not only the study of the coherent bunch motion, but also of incoherent beam dynamics.

\section{A. Numerical model}

The tracking is based on beam transport by successive application of Courant-Snyder transfer matrices and "kicks". For this purpose, the ring is split into a discrete set of sections. The bunch is transported along each section by means of the transfer matrices according to the local Courant-Snyder parameters. At the end of each section the bunch interacts with an electron cloud (or any other source of impedance). The electron clouds are assumed to be 2D uniform rectangular distributions of electrons extending over 15-30 times the rms beam size depending on the beam energy. The bunch is sliced longitudinally and the interaction with the electron cloud takes place slice by slice. The computation of the bunch-electron-cloud interaction itself is performed using a particle-in-cell fastFourier-transform-type Poisson solver.

As the bunch passes through the electron cloud, the electrons are attracted towards the bunch and the cloud is pinched $[34,35]$. Snapshots of cloud pinches are shown in Fig. 13 for a cloud located in a straight section and a bending magnet. For the presented simulations, the beam is always initialized on axis. Slices towards the back are affected depending on the statistical properties of the slices towards the front which causes head-tail coupling [36].

Thus, the bunch-electron-cloud interaction can give rise to coherent bunch modes which under certain conditions may become unstable [36,37]. The coherent instability can be detected as an exponential growth of the emittance arising from statistical noise. As the emittance increases a saturation of the instability is observed. One of the reasons for this is the weakening of the electron cloud due to the emittance increase, which moderates the rise of the coherent instability.

This type of interaction may also induce incoherent emittance growth [8]. Incoherent emittance growth takes place linearly and starts out immediately. It can generate large tune spreads and promote interactions of single particles with the nonlinearities of the machine, but also with the intrinsically nonlinear electron-cloud itself by periodic crossing of resonances $[8,38]$.

The incoherent emittance growth should be interpreted with care, however [39]. It becomes dominant when either a single interaction is highly pronounced or when the integrated force of all interactions over one turn

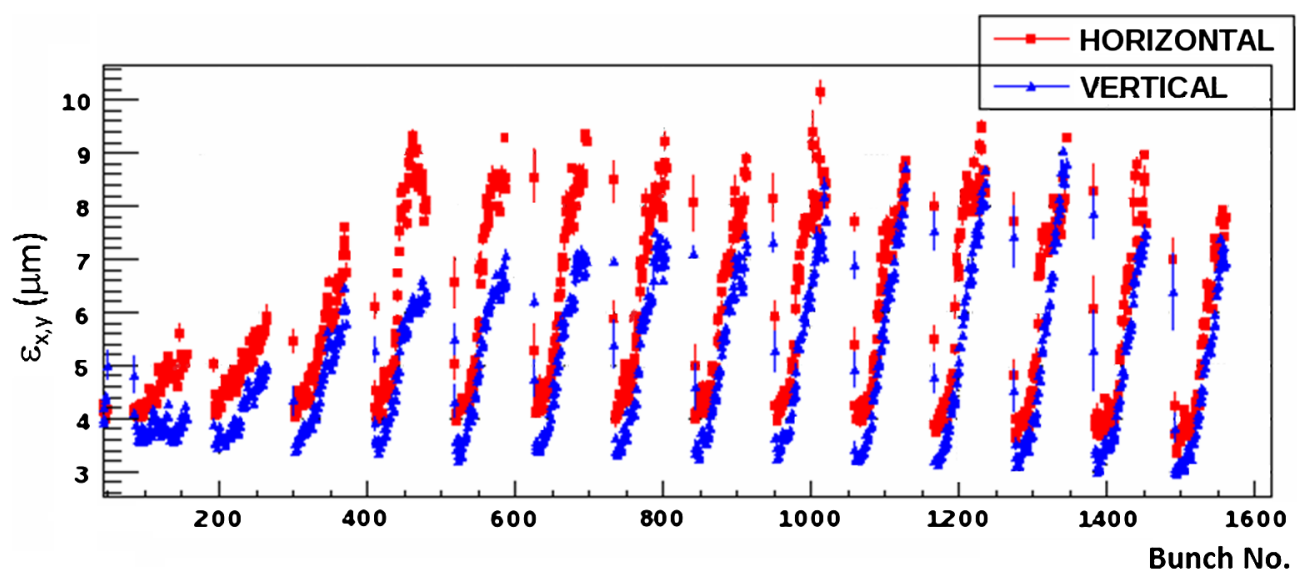

FIG. 12. Bunch-by-bunch normalized horizontal and vertical rms emittance in units of micron along multiple successive bunch trains measured by the gated synchrotron-light monitor. 

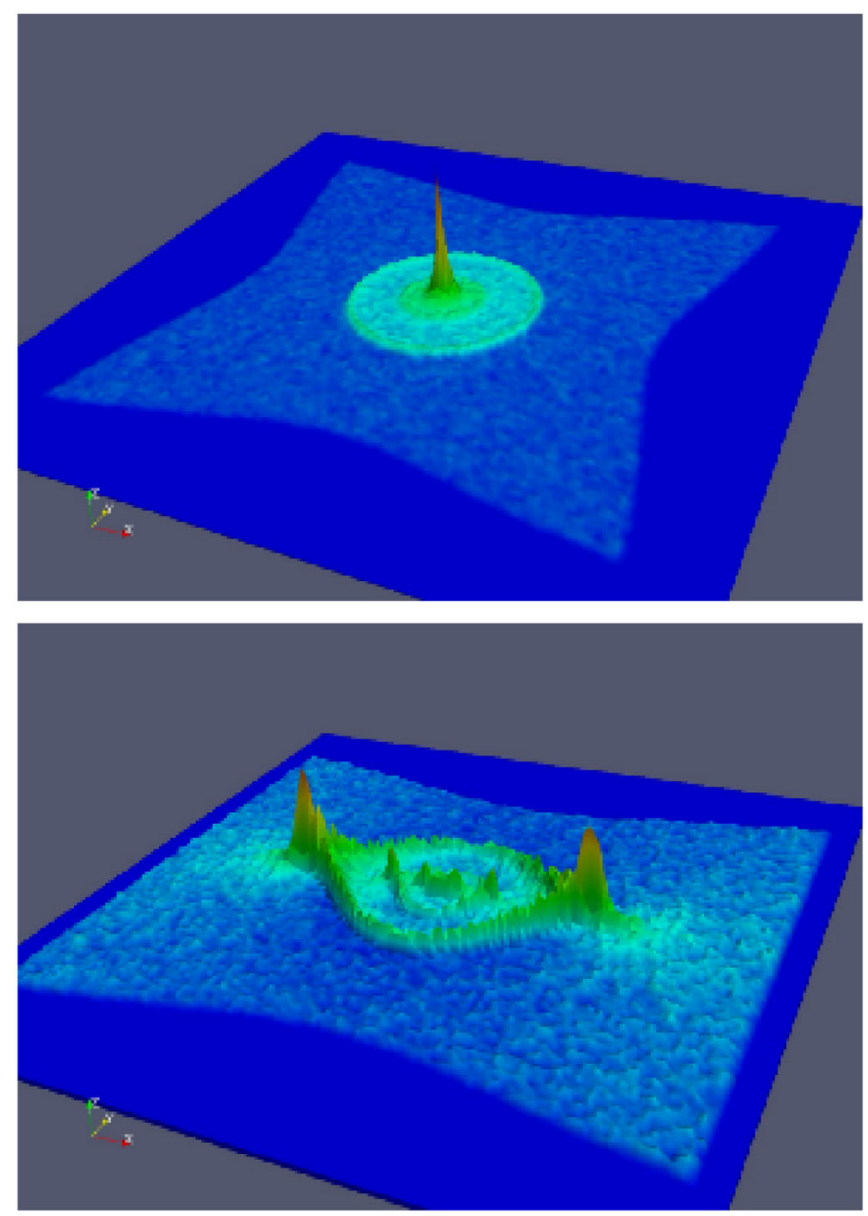

FIG. 13. Electron-cloud pinch snapshot during passage of a beam in a straight section (top) and a bending magnet (bottom).

exceeds a certain threshold. In both cases the tune spread and the resulting incoherent emittance growth become so strong that any coherent effect is masked. Since the distribution of the electron clouds along the ring is not known, the resonances excited in the simulation can be rather artificial. Nevertheless, the shape of the tune footprint gives an indication on what resonances might be crossed and can become a potential source of emittance degradation.

In the following we report simulation results for either bending magnets or field-free straight sections, assuming that the entire ring consists of only one or the other. The true instability growth rate in the LHC would be approximately the weighted sum of these two contributions:

$$
\frac{1}{\tau} \approx 0.66 \frac{1}{\tau_{\text {bend }}}+0.34 \frac{1}{\tau_{\text {straight }}},
$$

where 0.66 and 0.34 represent, roughly, the fraction of the circumference occupied by bending magnets, and the rest, respectively. The total tune spread should be computed in a similar way as a weighted sum.

\section{B. Coherent emittance growth}

To study the threshold values for coherent instabilities both at $450 \mathrm{GeV}$ and at $3.5 \mathrm{TeV}$, the central cloud density was scanned for different values of the bunch intensity and of the transverse emittance. The emittance evolution is given by the superposition of the stationary solution and the coherent mode as

$$
\varepsilon_{n}(t)=\varepsilon_{0}+a \exp (-i \Delta \Omega t),
$$

with the complex tune shift $\Delta \Omega$. From this we define the rise time as

$$
\tau=\frac{1}{\operatorname{Im}(\Delta \Omega)}
$$

The instability threshold was set at rise times below $10 \mathrm{~ms}$, which correlates well with the evolution of the emittance.

We first look at the results for a straight section. Figure 14 shows a typical scan of the central cloud density at flat-bottom at nominal intensity and at a transverse normalized emittance of $2.5 \mu \mathrm{m}$. The emittance
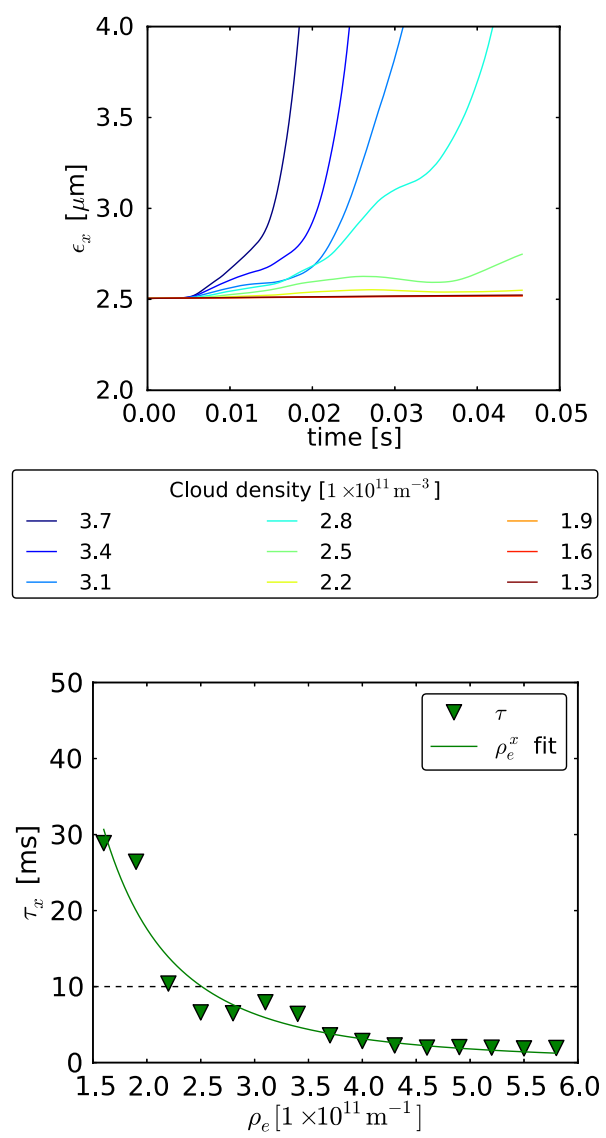

FIG. 14. Top: Emittance evolution for different central cloud densities at an energy of $450 \mathrm{GeV}$ considering a proton beam passing through a straight section. The legend entries indicate the central cloud densities in $1 \times 10^{11} \mathrm{~m}^{-3}$. Bottom: The corresponding rise time values with threshold set at $10 \mathrm{~ms}$. 

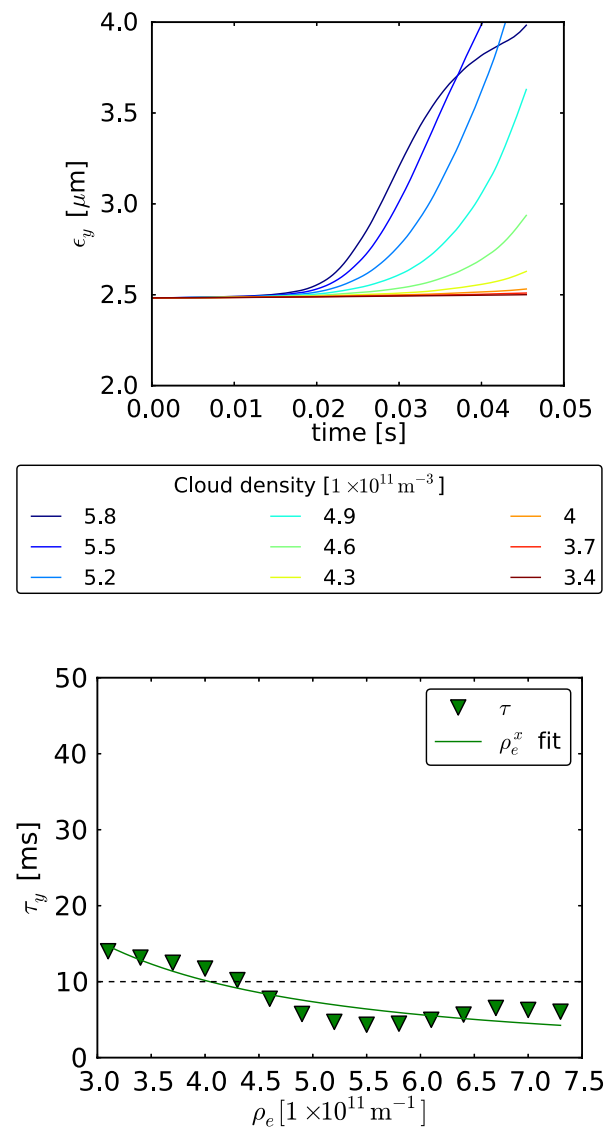

FIG. 15. Top: Emittance evolution for different central cloud densities at an energy of $3.5 \mathrm{TeV}$, considering a proton beam passing through a straight section. The legend entries indicate the central cloud densities in $1 \times 10^{11} \mathrm{~m}^{-3}$. Bottom: The corresponding rise time values with threshold set at $10 \mathrm{~ms}$.

curves are plotted on the top graph together with the corresponding rise time values on the bottom graph. The dependency of the rise times versus the central cloud density for this particular case is best approximated by

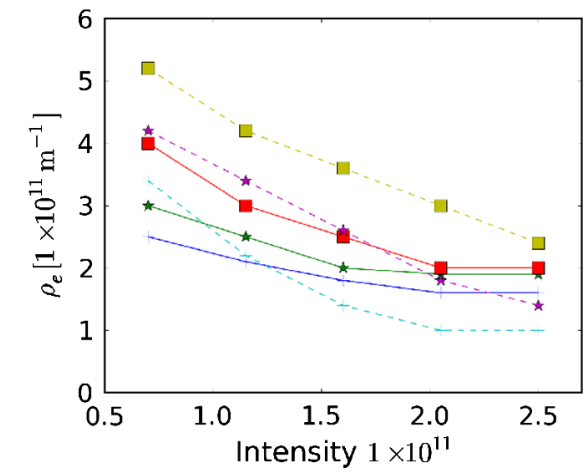

\begin{tabular}{|lc}
$-450 \mathrm{GeV}-1.50 \mu \mathrm{m}$ & $-+-3.5 \mathrm{TeV}-1.50 \mu \mathrm{m}$ \\
$\rightarrow-450 \mathrm{GeV}-2.50 \mu \mathrm{m}$ & $-\star-3.5 \mathrm{TeV}-2.50 \mu \mathrm{m}$ \\
$-450 \mathrm{GeV}-3.75 \mu \mathrm{m}$ & $-\square-3.5 \mathrm{TeV}-3.75 \mu \mathrm{m}$ \\
\hline-45
\end{tabular}

FIG. 16. Instability threshold values for the straight section for $450 \mathrm{GeV}$ and for $3.5 \mathrm{TeV}$ and different transverse emittances.
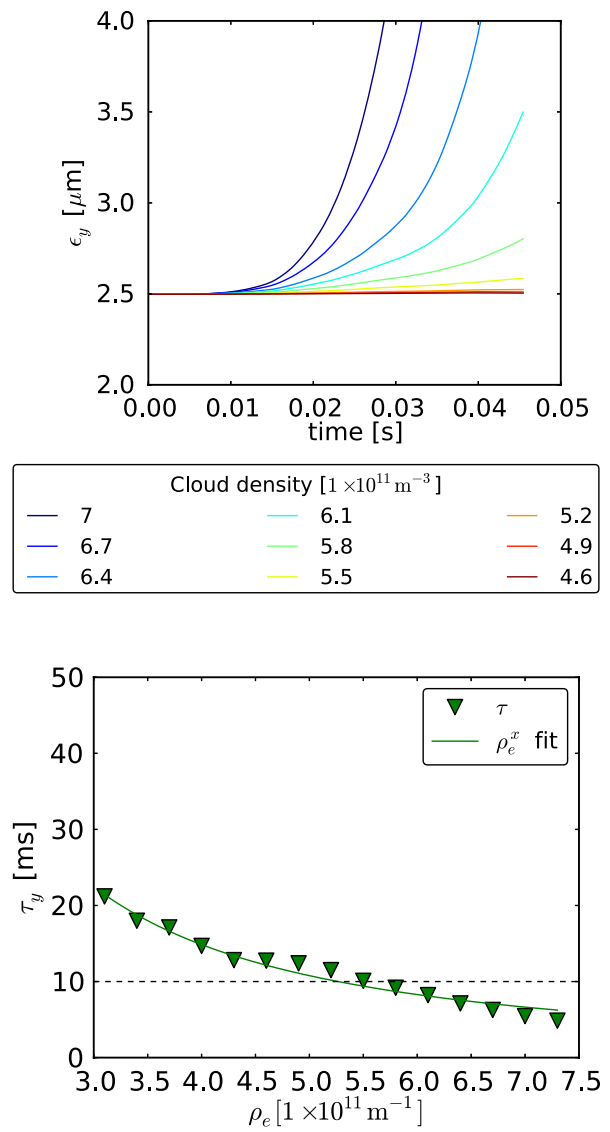

FIG. 17. Top: Emittance evolution for different central cloud densities at an energy of $450 \mathrm{GeV}$, considering a proton beam passing through a bending field. The legend entries indicate the central cloud densities in $1 \times 10^{11} \mathrm{~m}^{-3}$. Bottom: The corresponding rise time values with threshold set at $10 \mathrm{~ms}$.

$\tau \sim \rho_{e}^{-2.49}$. At low central densities the rise times are in the order of several seconds indicating that a coherent emittance growth is likely to be damped by other mechanisms.

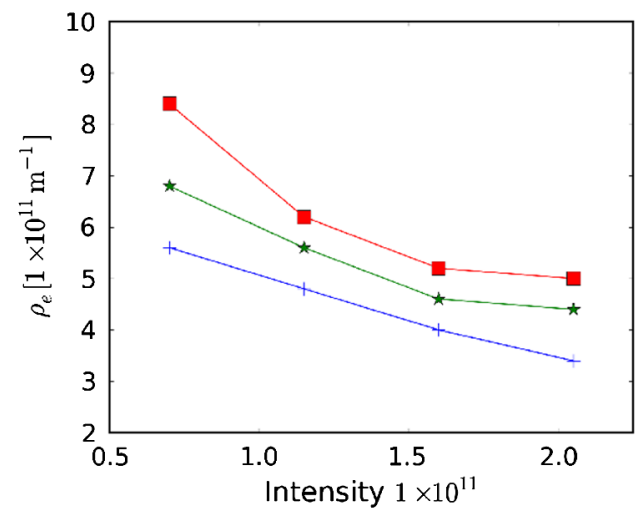

$450 \mathrm{GeV}-1.50 \mu \mathrm{m}$

* $450 \mathrm{GeV}-2.50 \mu \mathrm{m}$

$-450 \mathrm{GeV}-3.75 \mu \mathrm{m}$

FIG. 18. Instability threshold values for the bending magnets at $450 \mathrm{GeV}$ and different transverse emittances. 

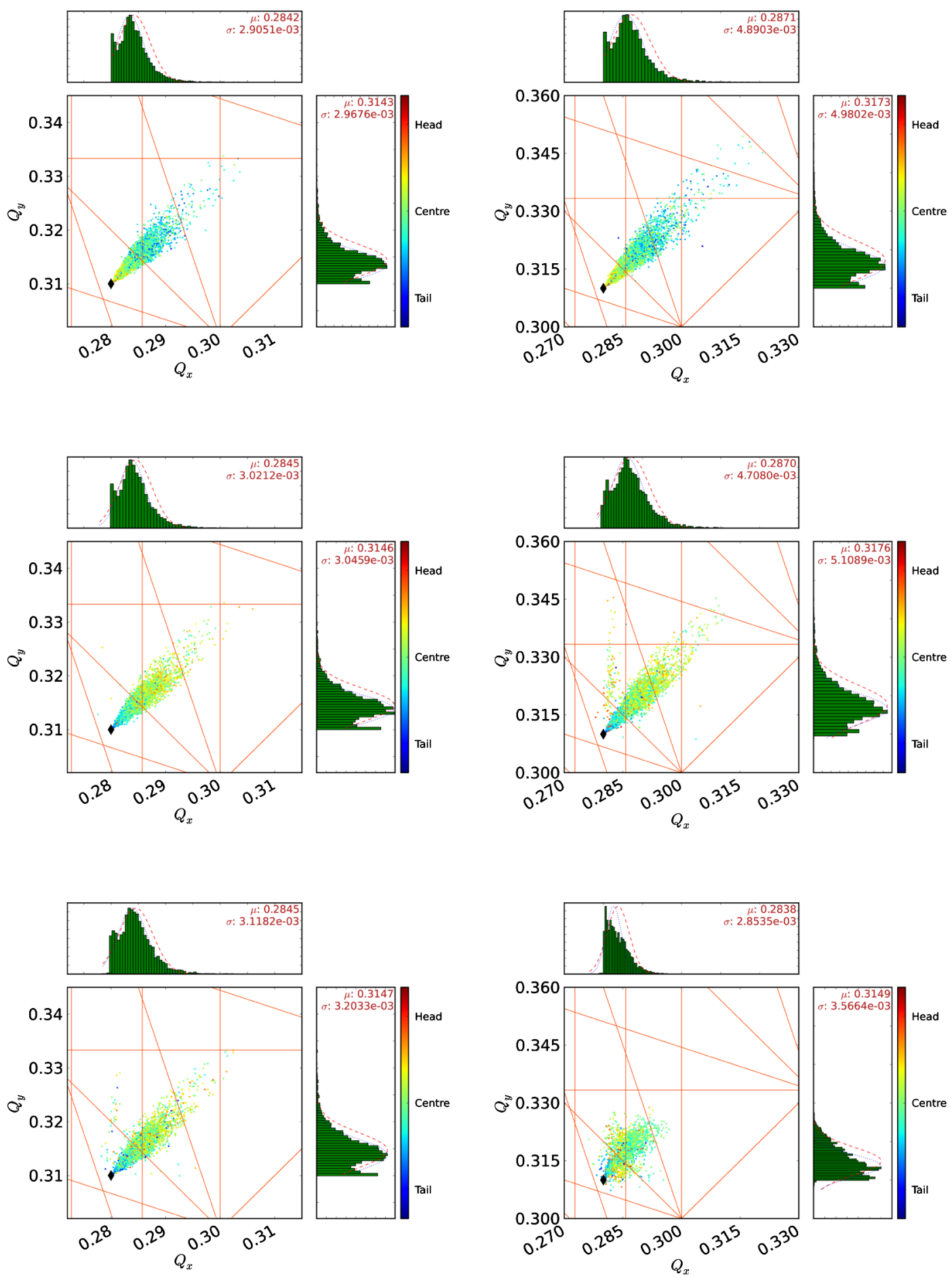

FIG. 19. Tune footprint snapshots in a straight section at $450 \mathrm{GeV}$ after $1 / 6$ (top), $1 / 2$ (middle), and $3 / 2$ (bottom) synchrotron periods. Left: Below the instability threshold $\left(\rho_{c}=2.2 \times 10^{11} \mathrm{~m}^{-3}, n_{b}=1.15 \times 10^{11} \mathrm{ppb}\right)$. Right: Above the intensity threshold $\left(\rho_{c}=3.7 \times 10^{11} \mathrm{~m}^{-3}, n_{b}=1.15 \times 10^{11} \mathrm{ppb}\right)$. Resonance lines up to 11 th order have been included.

Figure 15 shows the same scan at flattop energy. At this energy the dependency of the rise times versus the central cloud density is approximated by $\tau \sim \rho_{e}^{-1.96}$. It is less steep than for the low-energy case and as a result, the instability threshold is shifted towards higher values. On the other hand, at low central densities the rise times are comparatively small and a coherent emittance growth emerges rather early. For both cases the coherent growth is clearly visible when the rise time decreases below $10 \mathrm{~ms}$.

Figure 16 summarizes the instability threshold values obtained for the straight section for both flat-bottom and 

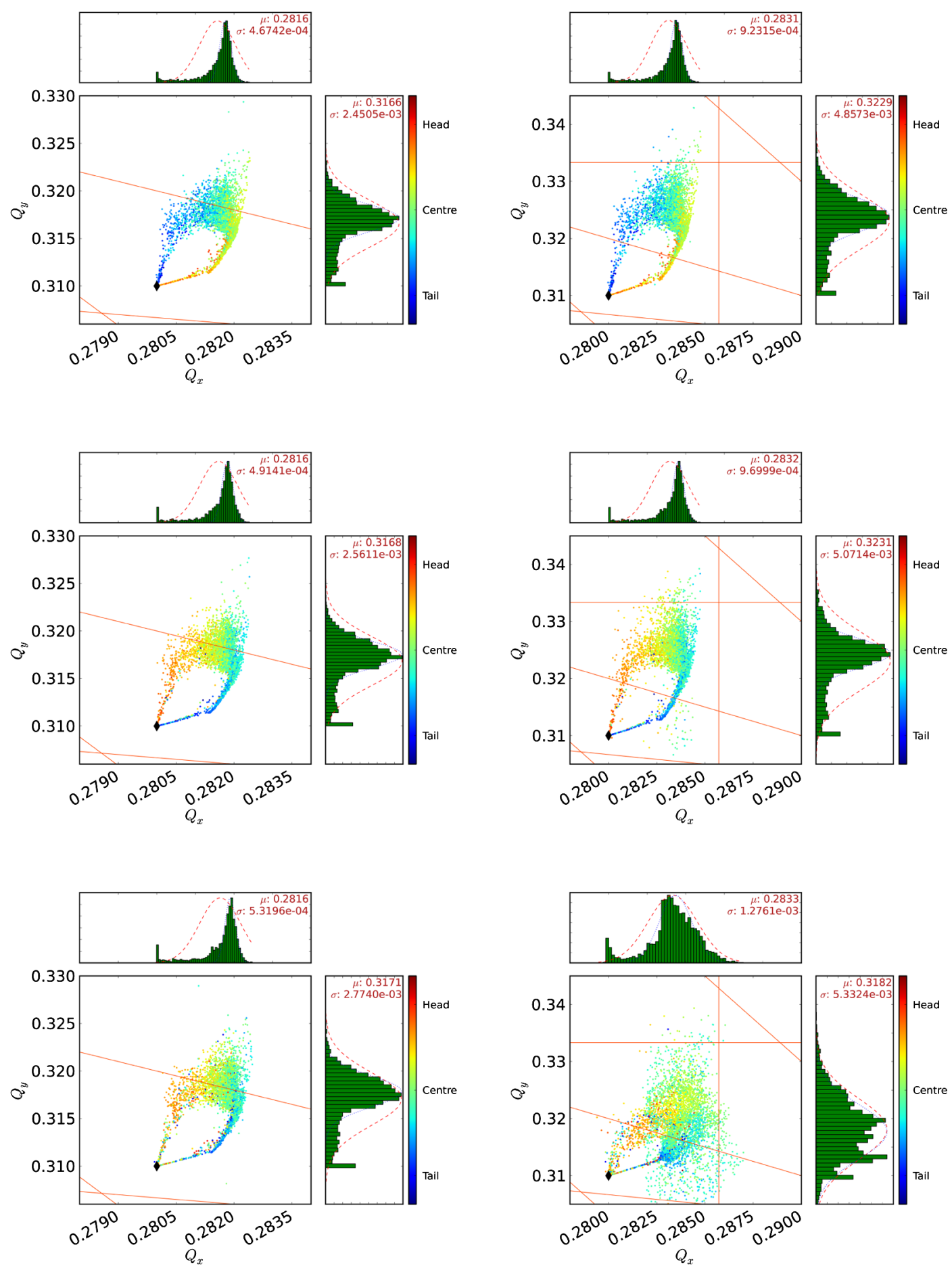

FIG. 20. Tune footprint snapshots in a bending magnet at $450 \mathrm{GeV}$ after $1 / 6$ (top), 1/2 (middle), and 3/2 (bottom) synchrotron periods. Left: Below the instability threshold $\left(\rho_{c}=4.3 \times 10^{11} \mathrm{~m}^{-3}, n_{b}=1.15 \times 10^{11} \mathrm{ppb}\right)$. Right: Above the intensity threshold $\left(\rho_{c}=8.5 \times 10^{11} \mathrm{~m}^{-3}, n_{b}=1.15 \times 10^{11} \mathrm{ppb}\right)$. Resonance lines up to 11 th order have been included.

flattop energy and different transverse emittances. As expected, the threshold values decrease together with the transverse emittances as a result of the reduced beam sizes. The dependency of the threshold on the bunch intensity is weak at low energy and becomes pronounced at higher energies. Thus, at low bunch intensities the thresholds start off higher at high energy. As the bunch intensity increases, the thresholds at high energy fall below the thresholds at low energy. In all cases the threshold values appear to saturate with increasing bunch intensity. This is simply due to the fact that the electron dynamics is dominated by the beam intensity and from a certain intensity onward 


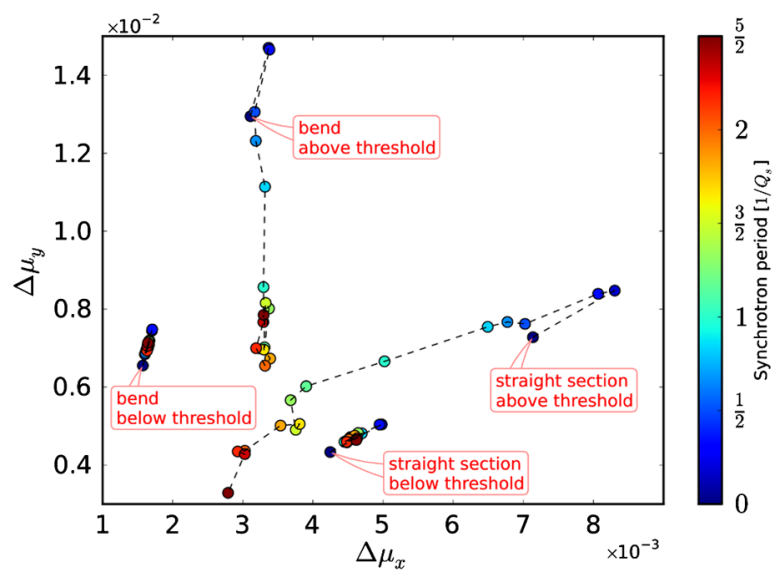

FIG. 21. Incoherent tune shift evolution at $450 \mathrm{GeV}$ and a transverse normalized emittance of $2.5 \mu \mathrm{m}$ for different cases.

all electrons in the vacuum pipe are pinched towards the beam. Therefore, the accumulated central cloud density during the bunch passage will no longer change and, hence, the threshold value for coherent instabilities no longer changes either.
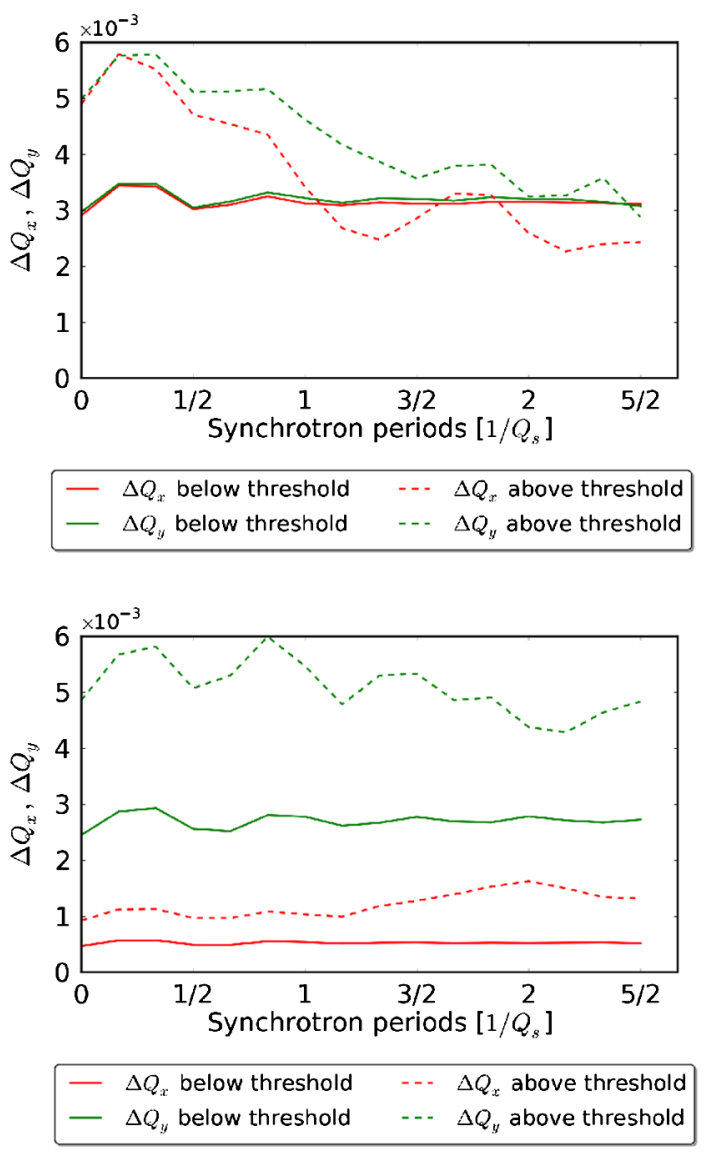

FIG. 22. Incoherent tune spread evolution at $450 \mathrm{GeV}$ and a transverse normalized emittance of $2.5 \mu \mathrm{m}$ in straight sections.
As opposed to the straight sections, in the bending magnets the electrons are forced to move along the magnetic field lines. Thus, their motion is restricted to the vertical plane. As a result, the impact of the cloud on the beam is strongly reduced in the horizontal plane and potential instabilities occur solely in the vertical plane.

Figure 17 again shows a typical scan of the central cloud density at flat-bottom at nominal intensity $(1.15 \times$ $10^{11} \mathrm{ppb}$ ) and at a transverse normalized emittance of $2.5 \mu \mathrm{m}$, for a bending magnet. The emittance curves are plotted in the top graph together with the corresponding rise time values in the bottom graph. The dependence of the rise times on the central cloud density is approximated by $\tau \sim \rho_{e}^{-1.44}$.

For the case of the bending magnets, at flattop no coherent instabilities were observed within the scan range of the central cloud density (up to $\rho_{c}=1.03 \times 10^{12} \mathrm{~m}^{-3}$ ).

Figure 18 summarizes the instability threshold values obtained for the bending magnets at injection energy. Again, the threshold values decrease together with the transverse emittances as a result of the reduced beam sizes. For the bunch intensities examined, typical threshold values in the straight sections are in the order of $2-4 \times$ $10^{11} \mathrm{~m}^{-3}$ at injection energy and $1-5 \times 10^{11} \mathrm{~m}^{-3}$ at 3.5 TeV. Threshold values in the bending magnets are of order 3-9 $\times 10^{11} \mathrm{~m}^{-3}$ at injection energy. Compared to the straight section, we observe that the threshold values for the bending magnets are roughly a factor 2 higher.

\section{Incoherent effects}

The electron-cloud potential is highly nonlinear and may lead to large tune spreads. Care must be taken to prevent the beam from crossing any lower order resonance lines in order to prevent incoherent emittance growth and to ensure long term stability. On the other hand, a large tune spread can also help stabilizing the beam.

To assess the incoherent tune shift and the tune spread imposed on the beam by the electron cloud, the tune footprint has been computed for different cases. The tune footprint evolution was determined at $450 \mathrm{GeV}$ and at $3.5 \mathrm{TeV}$ at a transverse normalized emittance of $2.5 \mu \mathrm{m}$ in the straight sections as well as in bending magnets. For all cases we have performed simulation studies below as well as above the coherent instability threshold to observe how the beam changes in tune space as it either experiences incoherent effects or undergoes a coherent instability.

Figures 19 and 20 show the tune footprint evolutions computed at $450 \mathrm{GeV}$ for straight sections and for bending magnets below (left plots) and above (right plots) the respective instability thresholds (at nominal bunch intensity, $1.15 \times 10^{11} \mathrm{ppb}$ ). Each tune footprint is obtained within a moving window that spans about a sixth of a synchrotron period. Particles are colored according to 

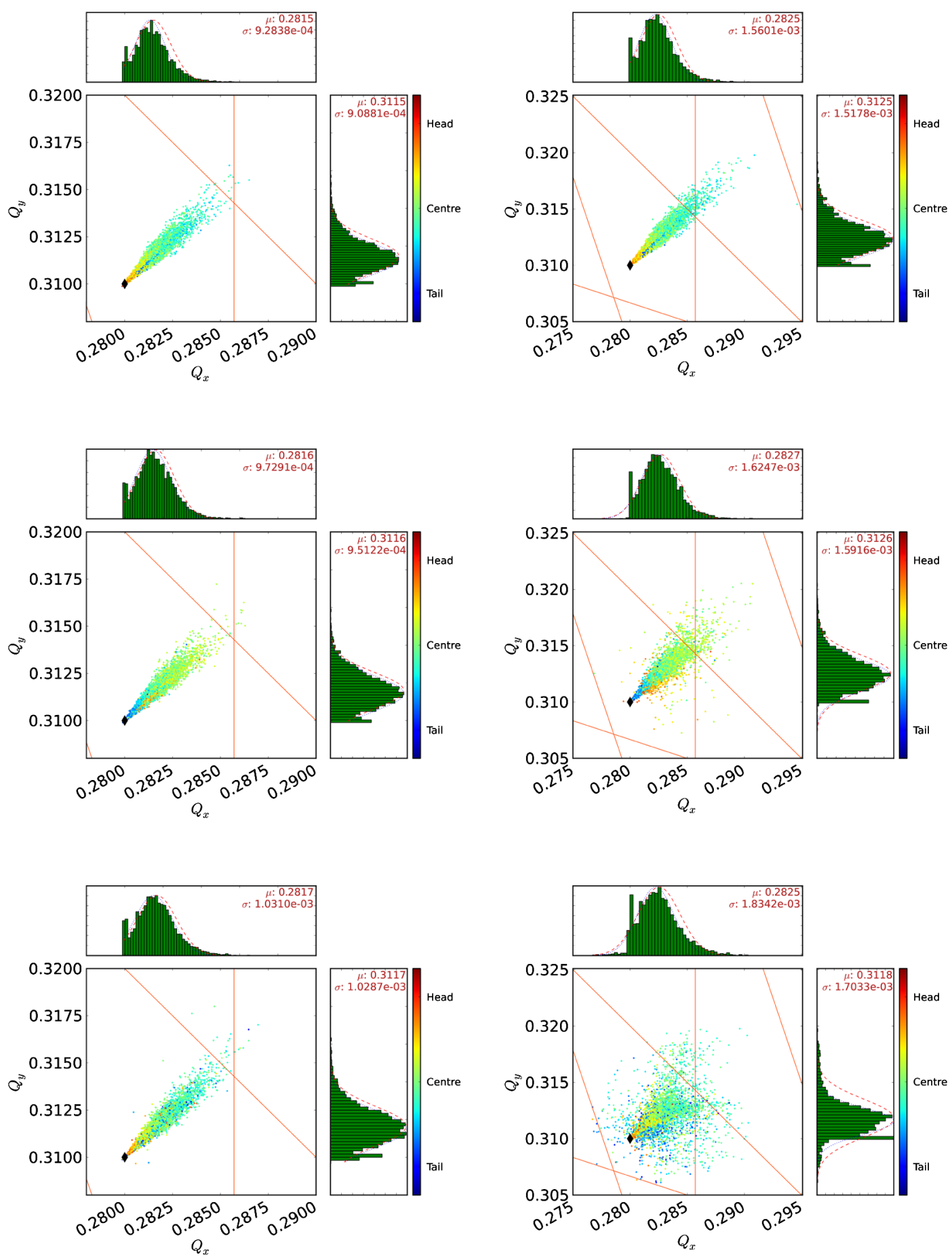

FIG. 23. Tune footprint snapshots in a straight section at $3.5 \mathrm{TeV}$ after $1 / 16$ (top), $1 / 2$ (middle), and 1 (bottom) synchrotron periods. Left: Below the instability threshold $\left(\rho_{c}=3.1 \times 10^{11} \mathrm{~m}^{-3}, n_{b}=1.15 \times 10^{11} \mathrm{ppb}\right)$. Right: Above the intensity threshold $\left(\rho_{c}=\right.$ $\left.5.2 \times 10^{11} \mathrm{~m}^{-3}, n_{b}=1.15 \times 10^{11} \mathrm{ppb}\right)$. Resonance lines up to 11 th order have been included.

their initial longitudinal positions along the bunch. Blue resembles particles towards the tail; red are particles towards the head of the bunch. It becomes evident how particles towards the tail of the bunch experience a larger tune shift. This is due to the increasing pinch accompanied by growing nonlinear fields as the bunch passes through the electron cloud. Along with this, the projections onto each axis are plotted as histograms. The statistical moments corresponding to the incoherent tune shift and tune spread are evaluated for each histogram and the corresponding Gaussian curve is sketched in red just for orientation. 


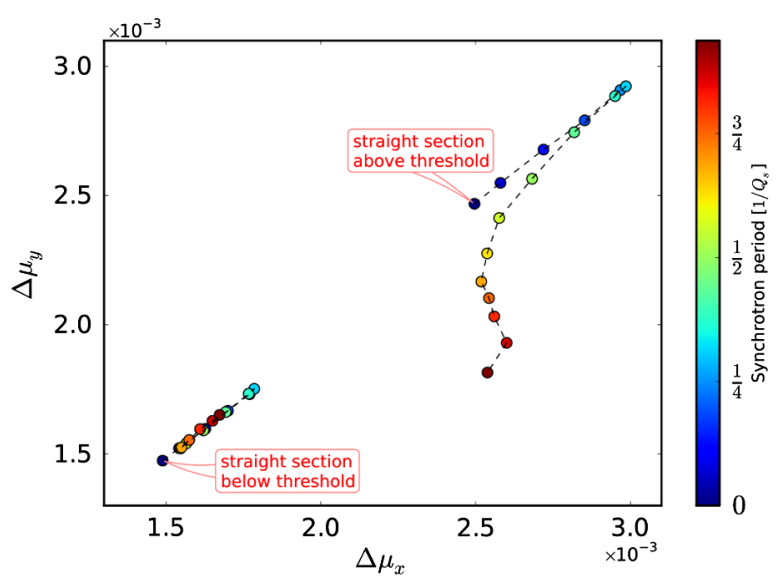

FIG. 24. Incoherent tune shift evolution at $3.5 \mathrm{TeV}$ and a transverse normalized emittance of $2.5 \mu \mathrm{m}$ for different cases.

Figure 21 shows the evolution of the incoherent tune shift in both planes over different fractions of a synchrotron period. Apart from giving absolute estimates on the incoherent tune shift, Fig. 21 also points out the different behavior below and above the instability threshold. Below the threshold the incoherent tune shift tends to oscillate before reaching a new equilibrium value while above the threshold the tune shift starts up at a large value and quenches as the beam undergoes an instability, thus smearing a range of

$$
\sqrt{\Delta \mu_{x}^{2}+\Delta \mu_{y}^{2}} \approx 7.5 \times 10^{-3}
$$

in tune space. It also becomes apparent how within a bending magnet the incoherent tune shift takes place predominantly in the vertical plane. This is a result of the symmetry of the pinch where the electric fields tend to cancel in the horizontal plane while they add up in the vertical plane. The rms tune spread evolution depicted in Fig. 22 shows a similar behavior.

Figure 23 shows the tune footprint evolutions computed at $3.5 \mathrm{TeV}$ for straight sections only since for the bending magnets at this energy no coherent instabilities were observed within the scan range of the electron-cloud density $\left(1.3 \times 10^{12} \mathrm{~m}^{-3}\right)$. The tune footprints are plotted below (left plots) and above (right plots) the respective instability thresholds. Each tune footprint is obtained within a moving window that spans about a 16th of a synchrotron period.

The general tune footprint evolution is similar to the low-energy case. However, compared to the low-energy case the beam appears to be more rigid in tune space. The overall incoherent tune shifts are roughly about a factor $8 / 3$ lower. From Fig. 24, below the threshold the incoherent tune shift still tends to oscillate before reaching a new equilibrium value. Above the threshold the tune shift again quenches as the beam undergoes an instability, now smearing a range of just

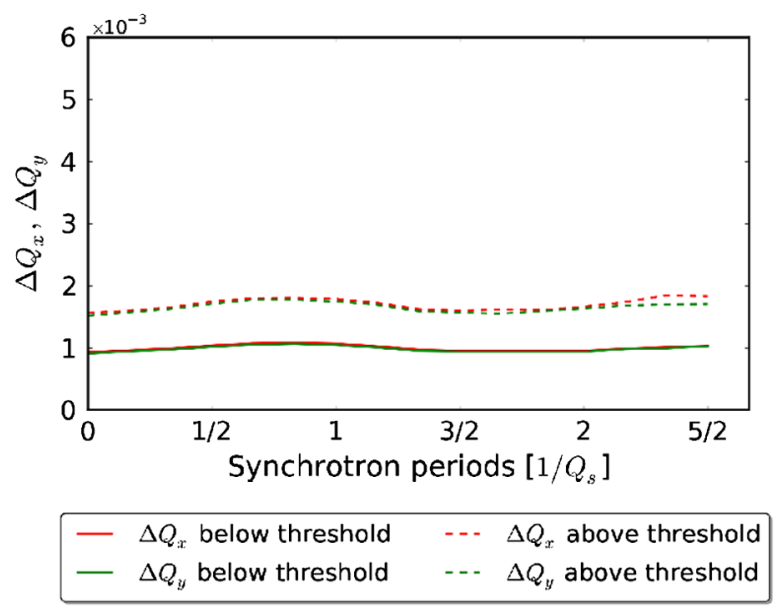

FIG. 25. Incoherent tune spread evolution at $3.5 \mathrm{TeV}$ and a transverse normalized emittance of $2.5 \mu \mathrm{m}$ in straight sections (top) and in bending magnets (bottom).

$$
\sqrt{\Delta \mu_{x}^{2}+\Delta \mu_{y}^{2}} \approx 1.5 \times 10^{-3}
$$

in tune space. The rms tune spread shown in Fig. 25 remains nearly constant and compared to the low-energy case it is reduced by about a factor of 3 .

Summarizing, the electron-induced tune spreads at nominal bunch intensity and injection energy are of the order of $3 \times 10^{-3}$ just below the instability threshold $\left(\rho_{c} \approx 2.2 \times 10^{11} \mathrm{~m}^{-3}\right)$ in the straight section and about 3 times lower at $3.5 \mathrm{TeV}$. In the case of bending magnets, this value is of the order of $2.5 \times 10^{-3}$ just below the instability threshold $\left(\rho_{c} \approx 4.3 \times 10^{11} \mathrm{~m}^{-3}\right)$.

\section{LHC CONDITIONING STATUS AND GOALS}

In 2010 and 2011 first electron-cloud effects have been observed with proton beams in the LHC. Rapid surface conditioning has allowed reducing the bunch spacing from $150 \mathrm{~ns}$ over $75 \mathrm{~ns}$ down to $50 \mathrm{~ns}$, already during 2010 . In order to reach the design LHC bunch spacing of $25 \mathrm{~ns}$ in physics operation, further conditioning of the secondary emission yield is required.

Thanks to the benchmarking of either vacuum or heatload observations against simulations, described in this paper, we have been able to follow up the evolution of $\delta_{\max }$ during machine conditioning, in both the warm straight sections and the cold arcs of the LHC. Figure 26 summarizes the approximate time evolution of $\delta_{\max }$ in the "warm-warm" transition regions where pressure gauges are located as well as in the arc dipoles. The multipacting thresholds for 50-ns and 25-ns bunch spacing are also indicated in the figure. The strong scrubbing effect of the 25-ns beam during the machine development (MD) sessions is evident. Only ca. $35 \mathrm{~h}$ in total was spent with this beam in these sessions, without which secondary emission yield values below the 50-ns threshold would not have been reached. 


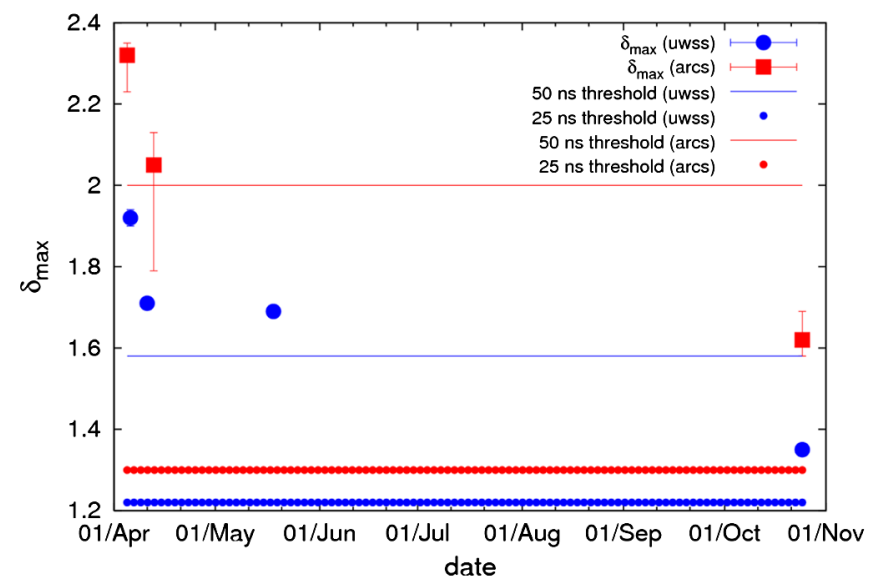

FIG. 26. Approximate time evolution (from April to October 2011) of $\delta_{\max }$ in the uncoated warm straight sections (chamber radius $40 \mathrm{~mm}$ ) as well as in the arc dipoles (chamber half apertures $22 \times 18 \mathrm{~mm}$ ) at the LHC at injection energy. The lowest values reachable with scrubbing correspond to the 25-ns multipacting threshold at $3.5 \mathrm{TeV}$. The first injection of a beam with 25-ns bunch-spacing beam took place on 29 June 2011. The error bars refer to different locations (in the case of straight sections they are included in the size of the point).

The evolution in the uncoated straight sections goes from an initial value of $\delta_{\max } \approx 1.9$ at the beginning of the scrubbing run in April 2011 to $\delta_{\max } \approx 1.35$ after the experiments with $25 \mathrm{~ns}$ were carried out. The points shown for 11 April and 19 May have been obtained by considering the average of the lines in Fig. 5 at $R=0.2$. In the case of the arc dipoles, we can observe as well an evolution towards lower values of $\delta_{\max }$ (from a value close to 2.3 to approximately 1.6 for the same period considered).

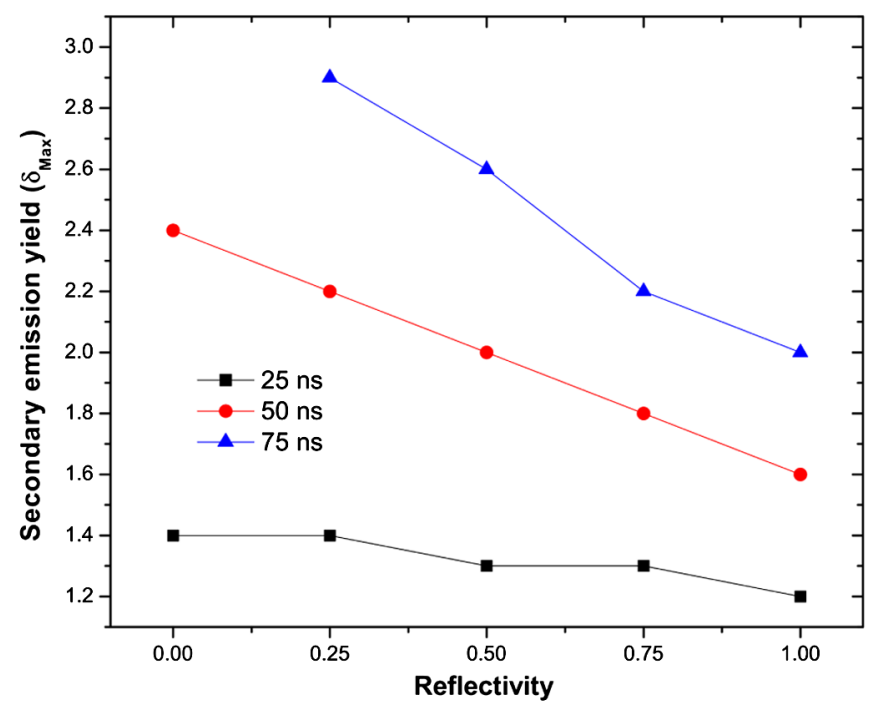

FIG. 27. Simulated multipacting thresholds for the LHC arc dipoles (chamber half apertures $22 \times 18 \mathrm{~mm}$, magnetic field of $8.33 \mathrm{~T}$, and an intensity of $1.15 \times 10^{11}$ protons per bunch) as a function of electron reflectivity for three different values of bunch spacing at $3.5 \mathrm{TeV}$ beam energy.
Figure 27 shows the calculated multipacting thresholds for different bunch-spacing values in the arc dipoles at $3.5 \mathrm{TeV}$ as a function of the reflectivity. For the present bunch spacing of $50 \mathrm{~ns}$, multipacting no longer occurs if the maximum secondary emission yield $\delta_{\max }$ decreases below about 2.0. This is perfectly consistent with observations in 2012 since no evidence of electron cloud is present anymore for $50 \mathrm{~ns}$ runs. At the 25 -ns bunch spacing a much lower $\delta_{\max }$ value below 1.3 is required to stop multipacting. This large threshold difference between 25 and 50 ns explains the strong electron-cloud related effects still observed during the MD sessions with the 25-ns bunch spacing, since the current $\delta_{\max }$ value is estimated to be larger, e.g., from our benchmarking of simulations against the measured arc heat load.
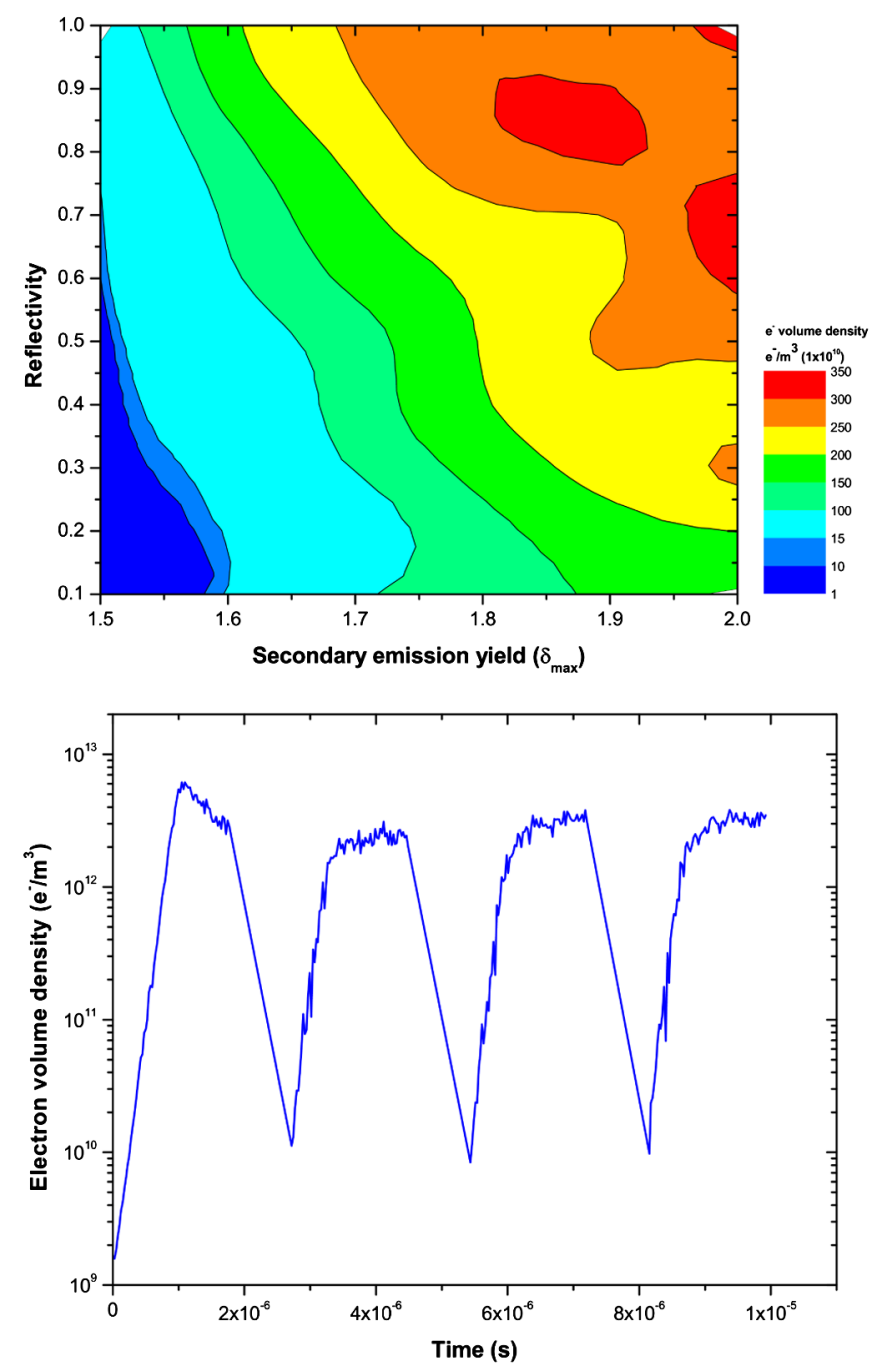

FIG. 28. Top: Contour plot of the electron volume density in a cylinder of $1 \mathrm{~mm}$ radius around the beam in a dipole magnet for the case with $25 \mathrm{~ns}$ bunch spacing at injection energy. Bottom: Time evolution of the simulated electron volume density (raw simulation data; $R=0.5, \delta_{\max }=1.8$ ). In the top picture the average density computed over the whole simulation time is shown. 
TABLE VI. Estimated values for $\delta_{\max }$ after the conditioning in 2011 and calculated threshold values.

\begin{tabular}{lcc}
\hline \hline & Uncoated straight sections & Arc dipoles \\
\hline Chamber aperture $(\mathrm{mm})$ & 40 (radius) & $22(x$ semiaxis $), 18(y$ semiaxis $)$ \\
Magnetic field $(\mathrm{T})$ & 0 & $0.535(450 \mathrm{GeV}), 4.2(3.5 \mathrm{TeV})$ \\
Estimated $\delta_{\max }$ & 1.35 & 1.62 \\
Threshold $\delta_{\max }(25 \mathrm{~ns}, 450 \mathrm{GeV})$ & 1.25 & 1.45 \\
Threshold $\delta_{\max }(25 \mathrm{~ns}, 3.5 \mathrm{TeV})$ & 1.22 & 1.3 \\
Threshold $\delta_{\max }(50 \mathrm{~ns}, 450 \mathrm{GeV})$ & 1.63 & 2.1 \\
Threshold $\delta_{\max }(50 \mathrm{~ns}, 3.5 \mathrm{TeV})$ & 1.58 & 2.0 \\
\hline \hline
\end{tabular}

Figure 28 (top) presents a contour plot of the electron volume density for a dipole section (corresponding to simulation set $\mathrm{C}$ described earlier). The instability threshold in the dipole magnets is around $3 \times 10^{11} \mathrm{~m}^{-3}$. From the figure this means that considering $R \approx 0.5$ we need a $\delta_{\max } \approx 1.55$ to avoid instabilities. Figure 28 (bottom) shows the raw data from the simulations for the case $R=$ 0.5 and $\delta_{\max }=1.8$. The central volume density is computed inside a transverse circle of 1-mm radius. Then we take the average over the total simulation time.

Table VI summarizes the estimations to date together with the calculated threshold values for $\delta_{\max }$. The latter would be the target values required to be reached in order to operate the LHC without any electron-cloud related limitation.

It is clear that further scrubbing is needed to achieve the target values. According to some estimates [40], approximately two weeks machine time would be required to achieve these values, since the scrubbing effect reduces with decreasing $\delta_{\max }$. Two weeks is too large a time period for the tight physics schedule planned for LHC in 2012, especially in view of the fact that 50 -ns beams offer a higher luminosity, owing to their smaller emittance and higher bunch intensities from the injectors. These considerations have yielded the decision of operating the LHC machine with 50-ns bunch spacing in 2012. Operating at 50-ns spacing slows down (or stops) the further surface conditioning and prevents approaching the target values. However, several beam-experiment periods have been scheduled for 2012 to further study the 25 -ns beams and to re-assess the conditioning state of the machine. Similar assessments and dedicated conditioning periods are expected to be needed in the future at the start of every LHC running period.

\section{CONCLUSIONS}

Various types of electron-cloud effects have been observed in the LHC for bunch spacings of $150 \mathrm{~ns}$ or below. The effects get much stronger for closer distances between bunches, but fast surface conditioning in 2010 and 2011 has made it possible to operate, in 2012, at a bunch spacing as small as $50 \mathrm{~ns}$ (twice the nominal) without any significant perturbation from electron cloud.
Novel methods to monitor the LHC surface conditioning due to the electron cloud itself, based on benchmarking simulations against experimental results, are under development and have been successfully applied to monitor the progress of surface conditioning in warm and cold regions of the LHC. The observable considered is either the pressure increase resulting from the electron cloud, which is taken to be proportional to the electron flux impinging on the vacuum-chamber walls, or the heat load deposited on the beam screen inside the cold magnets by the incident electron cloud, which can be directly measured by the cryogenics system (through the induced temperature increase of the helium passing through the beam-screen cooling capillaries).

By benchmarking the ratios of experimental pressures and of simulated electron fluxes for different beam configurations (e.g., for varying spacing between bunch trains or varying number of batches), we can pin down the value of the maximum secondary emission yield as well as the reflection probability for low-energy electrons. Applying this method to each of the different measurement sets available so far provides clear evidence for surface conditioning in the uncoated warm regions of the LHC, from an initial maximum secondary emission yield of about 1.9 down to about 1.35 , with $R \approx 0.2$, as can be seen in Fig. 26.

Similarly, in the cold arcs the observed heat loads can be benchmarked against the simulated energy deposition, showing a reduction of the secondary emission yield, in the arcs, from an initial value for the maximum secondary emission yield of about 2.3 down to about 1.6, with $R \approx$ 0.5, as also shown in Fig. 26.

In this paper we have furthermore compiled simulated multipacting thresholds, heat loads, and central electron densities for the LHC, considering different bunch spacings, filling patterns, and beam energies, as well as a large range of values for $\delta_{\max }$ and $R$.

These results can be used, in the future, to quickly estimate actual vacuum-chamber surface parameters $\left(\delta_{\max }\right.$ and $R$ ) from observations, as well as to predict the onset of multipacting-induced vacuum degradation or heat loads in the cold arcs (see Fig. 27).

In addition, emittance growth and coherent instabilities induced by the electron cloud in the LHC have been 
reviewed at both injection energy and at $3.5 \mathrm{TeV}$, independently for the straight sections and for bending magnets (assuming the entire machine to consist of either one or the other). In all cases, the instability threshold expressed in terms of electron density decreases for lower values of the transverse emittance. For the bunch intensities examined, typical threshold values in the bending magnets are of order 3-9 $\times 10^{11} \mathrm{~m}^{-3}$ at injection energy (for electron cloud in bending magnets no coherent instability was found at $3.5 \mathrm{TeV}$ over the parameter range considered), corresponding to $\delta_{\max } \approx 1.55$ (with $R=0.5$ ) at $25 \mathrm{~ns}$ bunch spacing. Threshold values in the bending magnets are at least a factor 2 higher than in the straight sections. Tune footprints below the instability threshold at injection energy reveal that the incoherent tune spread, of order a few times $10^{-3}$ near the instability threshold, is larger for the straight sections than for the bending magnets. In addition, for the bending magnets the incoherent tune spread is larger in the vertical plane, i.e., in the plane in which the electrons move.

Summarizing, electron-cloud effects have been observed in the LHC, are well reproduced in simulations, and mitigated by beam conditioning.

\section{ACKNOWLEDGMENTS}

The authors would like to thank V. Baglin, G. Bregliozzi, G. Iadarola, G. Lanza, M. Taborelli, L. Tavian, C. Yin-Vallgren, as well as all the machine operators for the support they provided with the machine operation and the experimental data, and for relevant and always enriching discussions. Part of the work presented here has been cofunded by the Mexican National Council on Science and Technology (CONACYT) and by the European Commission under the FP7 Research Infrastructures project EuCARDN, Grant Agreement No. 227579 (EuCARD-AccNet).

[1] F. Zimmermann, LHC Project Report No. 95, 1997.

[2] M. Izawa, Y. Sato, and T. Toyomasu, Phys. Rev. Lett. 74, 5044 (1995).

[3] K. Ohmi, Phys. Rev. Lett. 75, 1526 (1995).

[4] M. A. Furman, G. R. Lambertson, in Proceedings of the 10th European Particle Accelerator Conference, Edinburgh, Scotland, 2006 (EPS-AG, Edinburgh, Scotland, 2006), p. 1087.

[5] O. Gröbner, Vacuum 47, 591 (1996).

[6] O. Gröbner, in Proceedings of the 10th International Conference on High-Energy Accelerators (Protvino Institute for High Energy Physics, Protvino, USSR, 1977).

[7] F. Zimmermann, Phys. Rev. ST Accel. Beams 7, 124801 (2004).

[8] E. Benedetto, G. Franchetti, and F. Zimmermann, Phys. Rev. Lett. 97, 034801 (2006).

[9] W. Fischer et al., Phys. Rev. ST Accel. Beams 11, 041002 (2008).
[10] J. M. Jimenez, G. Arduini, P. Collier, G. Ferioli, B. Henrist, N. Hilleret, L. Jensen, K. Weiss, and F. Zimmermann, in Proceedings of ECLOUD'02 (CERN, Report No. CERN-2002-001, 2002), p. 17.

[11] J. M. Jimenez, G. Arduini, V. Baglin, G. Bregliozzi, P. Chiggiato, S. Claudet, G. Lanza, and L. Tavian, Report No. ATS/Note/2011/NNN (MD), 2011; in Proceedings of the Chamonix 2011 LHC Performance Workshop (Report No. CERN-2011-005, 2011), p. 56.

[12] G. Arduini et al., in Proceedings of the Chamonix 2011 LHC Performance Workshop, p. 63 (Ref. [11]).

[13] G. Rumolo et al., in Proceedings of IPAC2011, San Sebastián, Spain (EPS-AG, Spain, 2011), pp. 2862.

[14] G. Rumolo, G. Iadarola, O. Dominguez, G. Arduini, H. Bartosik, S. Claudet, J. Esteban-Müller, F. Roncarolo, E. Shaposhnikova, and L. Tavian, in Proceedings of the 3rd Evian Workshop on LHC Beam Operation, Evian-lesBains, France, 2011, edited by B. Goddard and S. Dubourg (CERN Report No. CERN-ATS-2012-083, 2012), p. 165.

[15] O. Brüning et al., in Proceedings of the 18th Particle Accelerator Conference, New York, 1999 (IEEE, New York, 1999), pp. 2629.

[16] R. Cimino, I. Collins, M. Furman, M. Pivi, F. Ruggiero, G. Rumolo, and F. Zimmermann, Phys. Rev. Lett. 93, 014801 (2004).

[17] The measured heat-load accuracy at the LHC is about $5-10 \mathrm{~mW} / \mathrm{m}$ [18]. By contrast, at RHIC and Tevatron the accuracy was 50 and $200 \mathrm{~mW} / \mathrm{m}$, respectively $[19,20]$.

[18] L. Tavian (private communication).

[19] W. Fischer et al., in Proceedings of the Mini Workshop on Electron Cloud Simulations for Proton and Positron Beams, pp. 63-68 (Report No. CERN-2002001, 2002).

[20] J.C. Theilacker (private communication).

[21] G. Rumolo and F. Zimmermann, Report No. CERN-SLNote-2002-036, 2002.

[22] G. Rumolo and F. Zimmermann, Phys. Rev. ST Accel. Beams 5, 121002 (2002).

[23] U. Iriso (unpublished).

[24] D. Schulte et al., in Proceedings of the 21st Particle Accelerator Conference, Knoxville, 2005 (IEEE, Piscataway, NJ, 2005), p. 1371.

[25] Several studies (e.g. [26]) reveal an evolution of the value of $\varepsilon_{\max }$ with the scrubbing process. This evolution depends on the scrubbing technique (either using an electron gun or a real beam) and several parameters such as the roughness of the surface, the previous surface treatment, the electrons energy, etc. It is clear that simulations depend on this parameter. So further investigation is needed to infer its evolution in the LHC.

[26] C. Yin Vallgren, Ph.D. thesis, Chalmers University of Technology, Göteborg , Sweden (CERN-THESIS-2011063, 2011).

[27] N. Hilleret et al., LHC Project Report No. 433, 2000.

[28] B. Henrist, N. Hilleret, C. Scheuerlein, and M. Taborelli, Appl. Surf. Sci. 172, 95 (2001).

[29] M. Belhaj, in Proceedings of the INFN-LNF/INFN-Pisa/ LER/EuCARD-AccNet Joint Workshop "ECLOUD'12," 
La Biodola, Isola d'Elba, Italy, 2012, edited by R. Cimino, G. Rumolo, and F. Zimmermann (to be published).

[30] I. Kaganovich, in Proceedings of the INFN-LNF/INFNPisa/LER/EuCARD-AccNet Joint Workshop "ECLOUD'12," La Biodola, Isola d'Elba, Italy, 2012, edited by R. Cimino, G. Rumolo, and F. Zimmermann (to be published).

[31] LHC Design Report No. CERN-2004-003-V-1, edited by O. S. Brüning, P. Collier, P. Lebrun, S. Myers, R. Ostojic, J. Poole, and P. Proudlock, 2004.

[32] V. Baglin, I. Collins, B. Henrist, N. Hilleret, and G. Vorlaufer, CERN LHC Project Report No. 472, 2002.

[33] M. A. Furman and M. Pivi, Phys. Rev. ST Accel. Beams 5, 124404 (2002).
[34] M.A. Furman and A.A. Zholents, in Proceedings of the Particle Accelerator Conference, Washington, DC, 1993 (IEEE, New York, 1993), pp. 1794-1796.

[35] E. Benedetto and F. Zimmermann, in Proceedings of the 9th European Particle Accelerator Conference, Lucerne, 2004 (EPS-AG, Lucerne, 2004), pp. 1834.

[36] K. Ohmi and F. Zimmermann, Phys. Rev. Lett. 85, 3821 (2000).

[37] K. Ohmi, F. Zimmermann, and E. Perevedentsev, Phys. Rev. E 65, 016502 (2001).

[38] G. Franchetti, I. Hofmann, W. Fischer, and F. Zimmermann, Phys. Rev. ST Accel. Beams 12, 124401 (2009).

[39] E. Benedetto, Ph.D. thesis, Politecnico Torino, 2006 (CERN-THESIS-2008-096, CARE-THESIS-06-007).

[40] G. Rumolo et al. (unpublished). 